\title{
Motion Compensation in High Resolution Synthetic Aperture Sonar (SAS) Images
}

\author{
R. Heremans, Y. Dupont and M. Acheroy \\ Signal and Image Center (SIC-RMA) \\ Belgium
}

\section{Introduction}

The sequence of echoes detected by an active Synthetic Aperture Sonar (SAS) is coherently added in an appropriate way to produce an image with greatly enhanced resolution in the azimuth, or along-track direction when compared with an image obtained from a standard Side Looking Sonar (SLS).

The SAS processing originates from the Synthetic Aperture Radar (SAR) concept. A complete introduction to SAR technique can be found in (Sherwin et al., 1962), (Walker, 1980), (Wiley, 1985) and (Curlander \& McDonough, 1991).

Raytheon was issued in 1969 a patent for a high-resolution seafloor imaging SAS (Walsh, 1969) and 1971 analyzed a potential system in terms of its resolution and signal-to-noise ratio. Cutrona was the first well-known radar specialist to point out how the various aspects of SAR could be translated to an underwater SAS (Cutrona, 1975). Hughes (1977) compared the performance of a standard SLS to an SAS and showed that the potential mapping rate for SAS was significantly higher than for side-looking sonar. At the time there was an assumption that the instability of the oceanic environment would prevent the formation of SAS imagery. Experimental work, which was performed by Williams (1976) and Christoff et al. (1982), refuted the instability worry. The verification of this assertion performed at higher frequencies by Gough \& Hawkins (1989). Later, other concerns regarding the stability of the towed platform were also raised and some rail- or wire-guided trails where set up to avoid this extra complication. Nowadays there are a multiple of systems as hull mounted SAS systems, towed SAS systems and Autonomous Underwater Vehicles (AUV) systems. For further reading one can find an extended historical background of SAS in (Gough \& Hawkins, 1997).

Time and experience were needed to adapt SAR algorithms to SAS systems; the SAS systems use smaller radiating elements in proportion to the wavelength, which leads to higher radiation pattern of SAS with respect to SAR. The range migration effect on synthetic aperture processing is significant and pronounced in SAS imagery. An additional difference between SAR and SAS systems is the synthetic aperture time being greater in one order of magnitude in SAS, which leads to a phase corruption due to the medium fluctuations and platform instabilities. Typical synthetic aperture times for SAR are of the order of seconds with a medium coherence of some days, whereas for SAS the typical synthetic aperture time is of the order of several minutes with a similar medium coherence time (Marx et al. 2000). 
In this chapter the theoretical background of SAS processing will be presented followed by the importance of motion compensation in high resolution imagery. To validate the accuracy of the motion estimation a simulator was developed in which different motion errors were applied. The quantification of the motion estimation was validated through the 2D Fourier space $(\omega, \mathrm{k})$-reconstruction algorithm.

\section{Processing requirements for SAS}

In order to explain profoundly (Bruce, 1992) the aspects of the synthetic aperture sonar technique, one has to introduce first the Side-Looking Sonar (SLS). The SLS is an imaging technique that provides two-dimensional reflectance maps of acoustic backscatter energy from the ocean floor. The maps are characterized by an intensity variation that is proportional to the acoustic backscatter signal strength (Somers, 1984). The geometry of the SLS is similar to the one of SAS. The systems ensonify strips on the seafloor using an acoustic projector that moves in a nominally straight line above the ocean floor with velocity $v$ as shown in Fig. 1. In this figure $\theta_{E}$ is the elevation beam width of the projector, $\theta_{H}$ is the azimuth beam width of the projector, $R_{S}$ is the instantaneous slant range measured from the sensor to a point on the seafloor, and $W$ is the one-sided ensonified ground-plane swath width. The reflectance map in SLS has coordinates of cross-track (measured in the slant plane) and along-track distances. The near-edge elevation ${ }^{1}$ angle $\theta_{e}$ is the elevation angle at minimum range, the elevation beam width and the height of the sonar with respect to the seafloor determine the size of the ensonified swath width. As the platform moves, a projector transmits acoustic pulses and a hydrophone listens for the echoes. The time delay of each echo provides a measure of slant range, while the ping-to-ping motion of the projector gives the along-track image dimension. As the seafloor is imaged, the data are recorded and represented in the slant range plane. In Fig. $1 . R_{\min }$ and $R_{\max }$ are the minimum and maximum slant ranges respectively and $d$ is the one-sided blind zone defined to be the no illuminated area directly below the platform (i.e. nadir).

\subsection{Slant range processing for SLS and SAS}

In SAS, along track and across track processing are completely disentangled. For both SLS and SAS systems, the slant range processing is identical and often also called across track processing. This processing is performed to obtain range information from the seafloor. Pulsed systems sense range by measuring the delay interval between the transmission and the reception of the pulse, as shown in the upper right figure of Fig. 1. Assuming range gates of duration $\tau$, the two seafloor objects $\mathrm{O} 1$ and $\mathrm{O} 2$ separated by $\Delta \mathrm{R}_{\mathrm{g}}$, will be resolved if their returns do not overlap in time. The round-trip travel time for a pulse associated with the object at range $R_{s}$ is given by;

$$
t=\frac{2 R_{s}}{c}
$$

and the incremental delay due to the proximate object $\mathrm{O} 2$ is;

1 The grazing angle $\theta_{\mathrm{g}}$ is given by the elevation angle $\theta_{\mathrm{e}}$ as: $\pi / 2-\theta_{\mathrm{e}}=\theta_{\mathrm{g}}$. 


$$
t+\tau=\frac{2\left(R_{S}+\Delta R_{s}\right)}{c}
$$

where $\mathrm{c}$ is the propagation speed in the medium. A measure of the slant plane separability is obtained by subtracting (1) from (2), and is given by

$$
\tau=\frac{2 \Delta R s}{c} .
$$

The relationship between ground-plane and slant-plane (see lower right figure of Fig. 1.) is approximated as

$$
\Delta R_{g}=\frac{\Delta R_{s}}{\cos \theta_{g}} .
$$

Therefore, two objects on the seafloor are fully resolvable if their ground separation satisfies

$$
\Delta R_{g} \geq \frac{c \tau}{2 \cos \theta_{g}}
$$
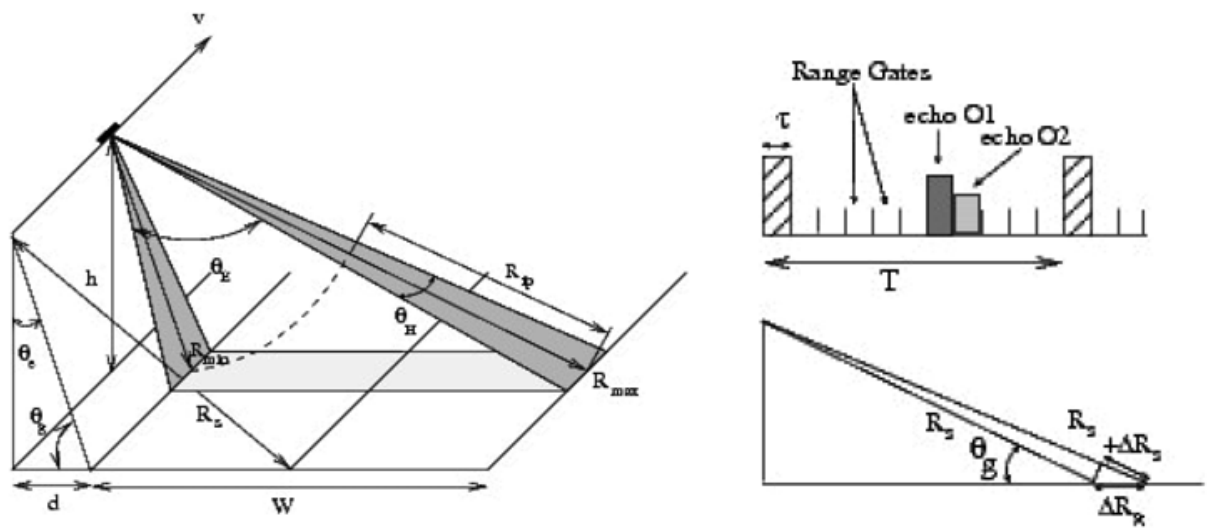

Fig. 1. Sonar geometry; (left) One-sided SLS geometry, (right) Time domain representation of a transmitted pulse and corresponding echoes.

Through equation (5) the range resolution is directly proportional to the ping duration $\tau$ and finer range resolution requires transmission of shorter pulses. However, in order to achieve very fine range resolution at long ranges, it may not be feasible to transmit very short pulses due to peak power limitation on the transmitter. In such cases, longer duration coded pulses can be transmitted with either frequency or phase modulation serving as the code. Upon arrival, the coded echo is decoded (compressed) so that the effective pulse length is significantly shorter in duration than the transmitted pulse (Stimson, 1983). The general relationship for range resolution $\rho_{r}$, is given by

$$
\rho_{r}=\frac{c \tau_{\text {eff }}}{2}=\frac{c}{2 B_{\text {eff }}}
$$


where $\tau_{\text {eff }}$ is the effective pulse length after compression and $B_{\text {eff }}$ represents the bandwidth of the compressed pulse.

The maximum unambiguous slant-range footprint can be determined by the effects of Pulse Repetition Interval (PRI) variations. If the PRI is set sufficiently large so that all echoes from a pulse are received prior to the transmission of the next pulse, there won't be an ambiguity. However if the PRI is decreased, the echoes from one pulse may not arrive at the receiver prior to the transmission of the following pulse. The minimum PRI value is equivalent to the requirement that the time between pulse transmission be greater than the time difference between the slant-range returns from the far- and near-edges of the slant-range footprint, $R_{f p}$. Thus the minimum acceptable PRI is,

$$
P R I_{\min }=\frac{2 R_{f p}}{c} .
$$

\subsection{Azimuth processing for SLS}

Fig. 2. shows the slant-plane view of an SLS with a projector of length D and azimuthal beam width $\theta_{H}$. The parameters $\delta_{a}^{\min }$ and $\delta_{a}^{\max }$ correspond to the linear azimuthal beam width at the minimum and maximum slant ranges respectively. The half-power angular beam width of a uniformly weighted rectangular aperture of length D is given in (Skolnik, 1980) by the approximate relationship

$$
\theta_{H} \cong \frac{\lambda}{D}
$$

where $\lambda$ is the acoustic wavelength of the signal. The resolution at slant-range distance $R_{s}$ of an SLS system is given by

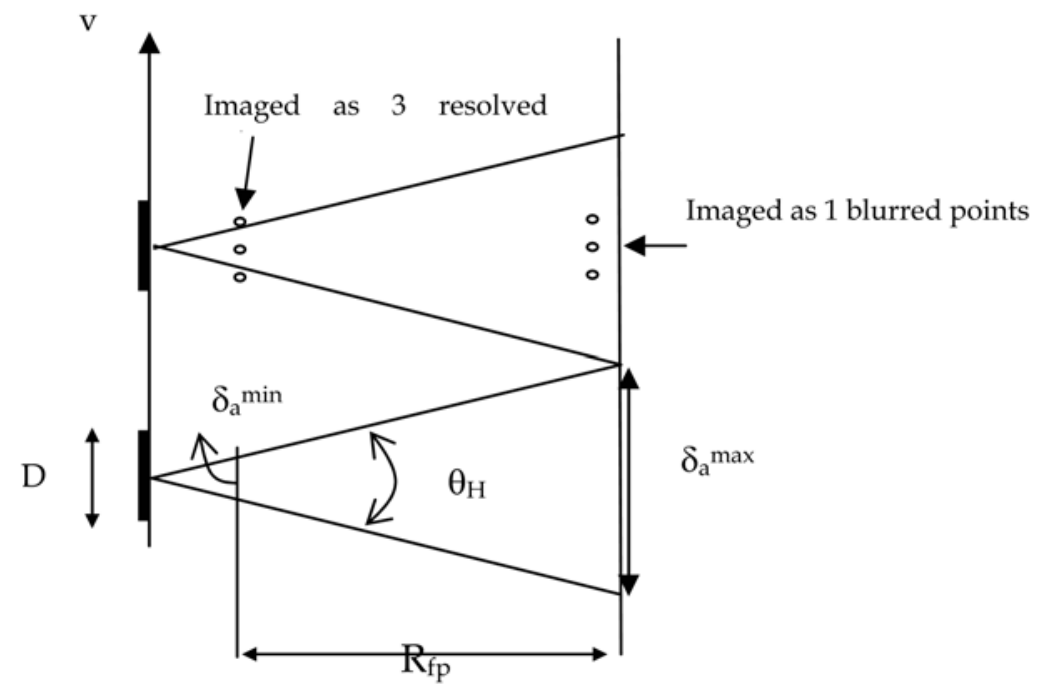

Fig. 2. Slant range, along track SASgeometry with beam spread as function of slant-range. Real-beam resolution degrading with increasing range 


$$
\delta_{a} \cong \frac{R_{s} \lambda}{D}
$$

In order to keep the resolution small as range increases, the frequency and/or the physicalaperture length $\mathrm{D}$ must be increased. The azimuth resolution however will ever stay dependent on slant-range.

In order to avoid along-track gaps in the image due to the beam pattern, one needs at least one sample for each along track resolution cell. Since the resolution cell width varies with range, the PRI selection will depend on the desired along-track resolution. Most side-scan systems are designed to realize the near-edge resolution

$$
P R I_{\max } \leq \frac{\delta_{a}^{\min }}{v}=\frac{\Delta_{a t}}{v}
$$

where $\delta_{a}^{\mathrm{min}}$ corresponds to the along-track sampling spacing $\Delta_{a t}$. In some cases, the alongtrack sampling spacing may be chosen finer than the azimuth resolution. This situation is called over sampling. If the lowest sampling rate while satisfying the Nyquist criterion is applied (called critical sampling) the along-track sample spacing is exactly equal to the azimuth resolution.

\subsection{Azimuth processing for SAS}

The physical aperture of a SAS system may be regarded as one element of a linear array extending in the direction of the platform motion as shown in Fig. 3. The SAS processing can than be compared to the combination of the individual receivers from the linear array into an equivalent single receiver. $L_{\max }$ is the maximum synthetic-aperture length possible for a given azimuth beam-width $\theta_{\mathrm{H}}$, while $\mathrm{L}_{\mathrm{act}}$ is the actual synthetic aperture length that may be shorter than $\mathrm{L}_{\max }$.

The azimuth resolution is obtained by sensing, recording, and processing the ping-to-ping phase history resulting from the variation in slant range caused by the projector's main lobe illumination pattern moving past seafloor scatterers. The maximum synthetic-array length is defined by the linear azimuth beamwidth at a given slant range $R_{s}, L_{\max }=R_{s} \theta_{H}$. The minimum effective horizontal beamwidth of the synthetic array is given by $\theta_{\min }=\lambda /\left(2 L_{\max }\right)$. The finest along track resolution that can be achieved from a focused synthetic aperture system is defined (Skolnik, 1980) as $\rho_{a}^{\min }=R_{s} \theta_{\min }=D / 2$.

\section{Necessity of motion compensation in SAS}

Following equation (7) Synthetic Aperture Sonar, suffers the upper bound on the pulse repetition frequency (PRF) imposed by the relatively slow sound speed in water. This in turn limits the platform velocity, and consequently introduces motion errors more easily due to the ocean instabilities like waves, water currents and wind. The effect of those motion errors on the SAS reconstruction will be exhibited in section 3.1.5. Motion compensation is the main key to obtain high-resolution SAS images, which are in turn indispensable to be able to perform not only reliable small target detection but also classification and identification. The prime concept solving the micronavigation issue is called Displaced Phase Centre Array (DPCA) that exploits in a unique way the spatial and temporal 
coherence properties of the seafloor backscatter in a multiple receiver array configuration. The DPCA concept will be explained and illustrated on simulated data in section 5 .

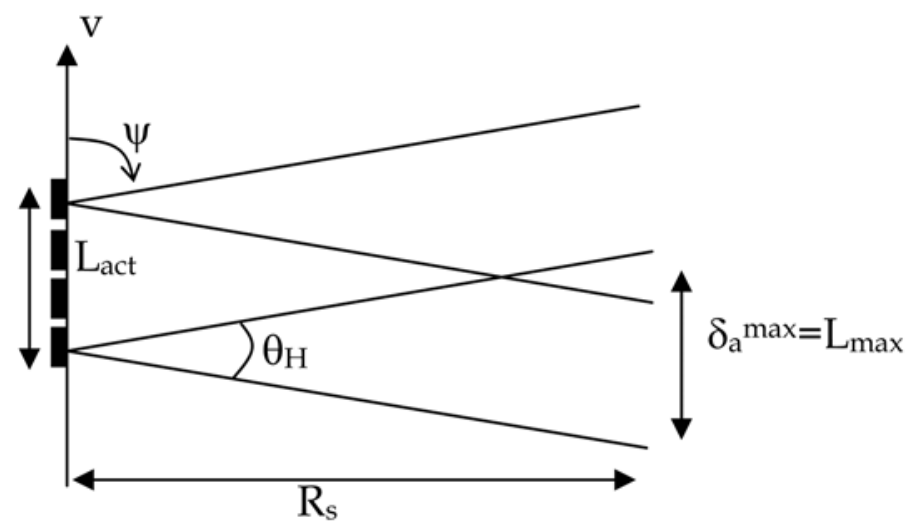

Fig. 3. Slant-plane view of an SLS with azimuthal beamspread $\theta_{\mathrm{H}}$. Real beam resolution degrades with increasing range $R_{s}$.

\subsection{Simulator}

The sonar simulator was designed to obtain the echo of a series of known point scatterers in a chosen scene. Let us consider first the case of a single transmitter/single receiver configuration.

\subsubsection{Single transmitter/ single receiver configuration}

Fig. 4 presents the 2D geometry of broadside strip-map mode synthetic aperture imaging systems. The surface imaged is substantially flat and has on top of it a collection of sub wavelength reflectors collectively described as the object reflectivity function $f f(x, y)$. Mostly $f f(x, y)$ is referred to as the object and consists of a continuous 2D distribution of omnidirectional (aspect independent) and frequency independent reflecting targets. This target area is illuminated by a side-looking sonar system travelling along a straight locus $u$ with a velocity $v$, moving parallel to the $y$-axis of the target area. The origins of the delay time axis $t$ and the range axis $x$ have been chosen to coincide. As the sonar platforms travels along $u$, it transmits a wide bandwidth phase modulated waveform $p_{m}(t)$ of duration $\tau$ seconds which is repeated every $T$ seconds. On reception, the coherent nature of the transmitter and receiver allows the echoes that have come from different pulses to be arranged into a $2 \mathrm{D}$ matrix of delay time $t$ versus pulse number. Since the platform ideally travels a constant distance between pulses, the pulse number can be scaled to position along the aperture $u$ in meters. Assuming the stop-start assumption, denoting that the sonar system does not move further along $u$ between the emission of the pulse and the respective reception of the signal, the strip-map system model represents the echoes detected at the output of the receiver and is approximately described by;

$$
e e_{m}(t, u) \approx \int_{x} f f(x, u) \otimes_{u}\left\{a(t, x, u) \otimes_{t} p_{m}\left(t-\frac{2}{c} \sqrt{x^{2}+y^{2}}\right)\right\} d x
$$


where $a(t, x, y)$ is the spatial-temporal response of the combined transmitter and receiver aperture. The output of the system given by a convolution in along-track, emphasize the two main problems faced by the inversion scheme which are:

- $\quad$ the system response is range variant

- there is a range curvature effect also called range migration.

Note that any function with a subscript $m$ implies modulated notation, i.e. the analytic representation of the function still contains a carrier term $\exp \left(i \omega_{0} t\right)$, where $\omega_{0}$ represents the carrier radian frequency. Demodulated functions are subscripted with a $b$ to indicate a base band function. The processing of base banded data is the most efficient method, as it represents the smallest data set for both the FFT (Fast Fourier Transform) and any interpolators. Many synthetic aperture systems perform pulse compression of the received reflections in the receiver before storage. The pulse compressed strip-map echo denoted by $s s_{m}$ is given by,

$$
s s_{m}(t, u)=p_{m}(t) \otimes_{t} e e_{m}(t, u) .
$$

The temporal Fourier transform of equation (12) is

$$
S s_{m}(\omega, u)=P_{m}(\omega) \mathfrak{I}_{t}\left\{e e_{m}(t, u)\right\}=P_{m}(\omega) E e_{m}(\omega, u)
$$

with the Fourier transform of the raw signal,

$$
E e_{m}(\omega, u)=P_{m}(\omega) \int_{x} \int_{y} f f(x, y) A(\omega, x, y-u) \exp \left(-i 2 k \sqrt{x^{2}+(y-u)^{2}}\right) d x d y
$$

with $\omega$ and $k$ the modulated radian frequencies and wave-numbers given by $\omega=\omega_{b}+\omega_{0}$ and $\mathrm{k}=\mathrm{k}_{\mathrm{b}}+\mathrm{k}_{0}$.

Throughout this chapter, radian frequencies and wave-numbers with the subscript 0 refer to carrier terms while radian frequencies and wave-numbers without the subscript $b$ refer to modulated quantities. At this point it is useful to comment on the double-functional notation and the use of the characters $e$ and $s$ like they are appearing in equations (11) till (14). The character $e$ is used to indicate non-compressed raw echo data, while $s$ is used for the pulse-compressed version (see equation (13)). Due to the fact that the echo is characterized by a $2 \mathrm{D}$ echo matrix (i.e. the scattering intensity as a function of azimuth and slant range) one needs a double-functional notation to indicate if a 1D Fourier transform, a 2D Fourier transform or no Fourier transform is applied on the respective variable. A capital character is used when a Fourier transform is applied. The first position of the doublefunctional notation refers to the slant-range direction (fast time) whereas the second position refers to the along-track direction (slow time). For example, $s S_{b}$ describes the pulsecompressed echo in the range/Doppler domain since a 1D Fourier transform is taken in the along-track domain. The subscript $b$ indicates that the pulse compressed echo data are also base banded. Putting the expression given in equation (14) into equation (13) leads to,

$$
\begin{aligned}
& S s_{m}(\omega, u)=\left|P_{m}(\omega)\right|^{2} \cdot \int_{x} \int_{y} f(x, y) \cdot A(\omega, x, y-u) . \\
& \exp \left(-i 2 k \sqrt{x^{2}+(y-u)^{2}}\right) d x d y .
\end{aligned}
$$

One can obtain the base banded version of equation (12) by taking the temporal Fourier transform on the base banded version of (14), 


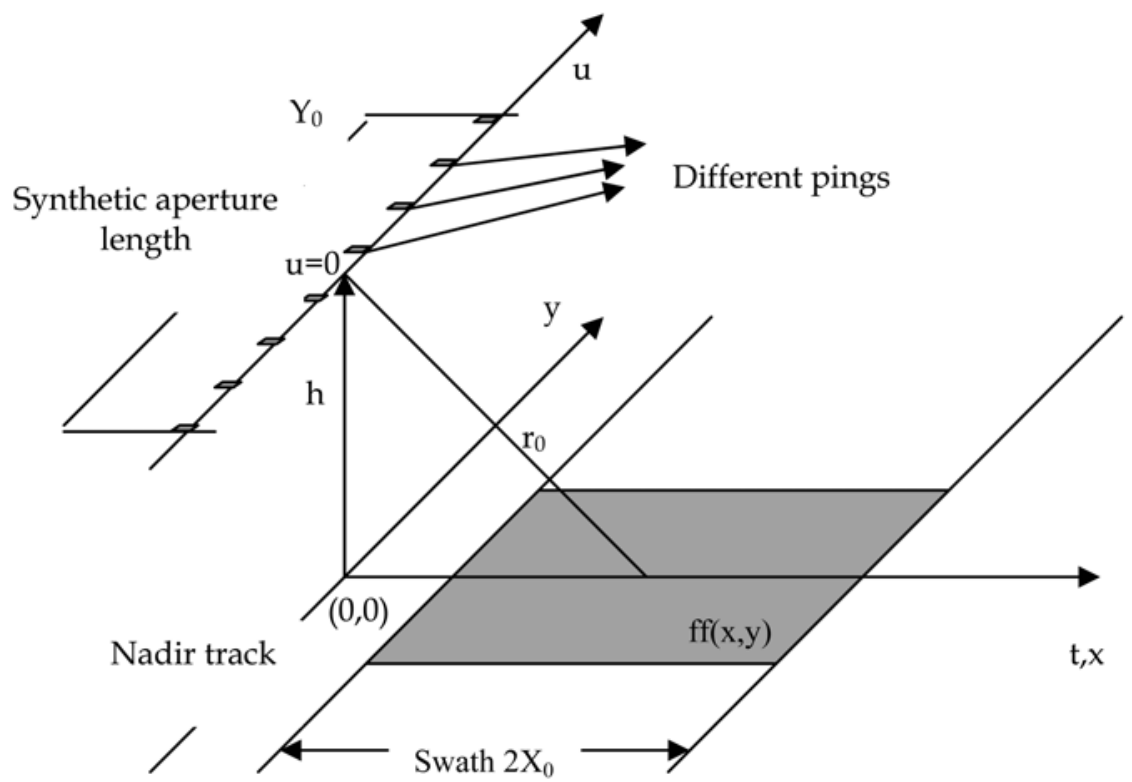

Fig. 4. Imaging geometry appropriate for a strip-map synthetic aperture system

$$
\begin{aligned}
& S s_{b}(\omega, u)=P_{b}\left(\omega_{b}\right) \cdot E e_{b}(\omega, u) \\
& \quad=\left|P_{b}\left(\omega_{b}\right)\right|^{2} \cdot \int_{x} \int_{y} f(x, y) \cdot A(\omega, x, y-u) \cdot \exp \left(-i 2 k \sqrt{x^{2}+(y-u)^{2}}\right) .
\end{aligned}
$$

The term $2 \sqrt{x^{2}+(y-u)^{2}}$ represents the travel distance from the emitter to the target and back to the receiver. In case of the start-stop assumption, the factor 2 appearing in front of the square root indicates that the travel time towards the object equals the one from the object back to the receiver. In the case the start-stop assumption is not valid anymore or in the case of multiple receivers, one has to split the term into two parts, one corresponding to the time needed to travel from the emitter to the target and one to travel from the target to the corresponding receiver. The above formulas, needed to build the simulator, will be extended in the following section towards the single transmitter multiple receiver configuration.

\subsubsection{Single transmitter/multiple receiver configuration}

The link with the single receiver can be made by reformulating equation (15) as follows,

$$
\begin{aligned}
E e_{m}(\omega, u)=\sum_{n} & P_{m}(\omega) \int_{x} \int_{y} f(x, y) A(\omega, x, y-u) . \\
& \quad \exp \left(-i k\left(R_{\text {out }}(u, n)+R_{\text {back }}(u, n, h)\right)\right) d x d y
\end{aligned}
$$

with $R_{\text {out }}(u, n)$ the distance from the transmitter to target $\mathrm{n}$ and $R_{\text {back }}(u, n, h)$ the distance from target $\mathrm{n}$ to the receiver $h$ for a given along-track position $u$. In the case of a multiple receiver array $R_{\text {out }}$ does not depend on the receiver number, 


$$
R_{\text {out }}(u, n)=\sqrt{x_{n}^{2}+\left(y_{n}-u\right)^{2}},
$$

whereas $R_{\text {back }}$ is dependent on the receiver number $h$, given by,

$$
R_{\text {back }}(u, n, h)=\sqrt{x_{n}^{2}+\left(y_{n}-u-h d_{h}\right)^{2}}
$$

with $d_{h}$ the along-track distance between two receivers. In the simulator the 3D echo matrix (depending on the along-track $u$, the return time $t$ and the hydrophone number $h$ ) will represent only a limited return time range corresponding with the target-scene. There is no interest in simulating return times where there is no object or where there is not yet a possibility to receive back signal scattered on the seafloor from the nearest range. Therefore the corresponding multiple receiver corresponding expression of equation (16) becomes,

$$
\begin{aligned}
E e(\omega, u, h)= & \sum_{n} P(\omega) \cdot \int_{x} \int_{y} f(x, y) \cdot A(\omega, x, y-u) . \\
& \exp \left(i k\left\{2 r_{0}-\left[R_{\text {out }}(n, u)-R_{\text {back }}(n, u, h)\right]\right\}\right) d x d y
\end{aligned}
$$

where the sum is performed over all $\mathrm{N}$ targets and $\mathrm{r}_{0}$ is the centre of the target-scene.

\subsubsection{Input signal $p(t)$}

The echo from a scene is depending on the input signal $p(t)$ generated by the transmitter and its Fourier transform $P(\omega)$. When a Linear Frequency Modulated (LFM) pulse $\mathrm{p}(\mathrm{t})$ is used it is expressed by,

$$
p_{m}(t)=\operatorname{rect}\left(\frac{t}{\tau}\right) \exp \left(i \omega_{0} t+i \pi K t^{2}\right)
$$

with $\omega_{0}(\mathrm{rad} / \mathrm{s})$ the carrier radian frequency and $K(\mathrm{~Hz} / \mathrm{s})$ the LFM chirp-rate. The rect function limits the chirp length to $t \in[-\tau / 2, \tau / 2]$. The instantaneous frequency is obtained by differentiation of the phase of the chirp,

$$
\omega_{i}(t)=\frac{d \phi(t)}{d t}=\omega_{0}+2 \pi K t
$$

This leads to a frequency of the input signal of ranging from $\omega_{0}-\pi \tau K$ till $\omega_{0}+\pi \tau K$, leading to a chirp bandwidth $B=K \tau$. Using the principal of stationary phase, the approximate form of the Fourier transform of the modulated waveform is

$$
P_{m}(\omega)=\operatorname{rect}\left(\frac{\omega-\omega_{0}}{2 \pi B}\right) \sqrt{\frac{i}{K}} \exp \left[-i \frac{\left(\omega-\omega_{0}\right)^{2}}{4 \pi K}\right] .
$$

The demodulated Fourier transform or pulse compressed analogue of $P_{m}(\omega)$,

$$
P_{c}(\omega)=P_{m}(\omega) \cdot P_{m}^{*}(\omega)=\frac{1}{K} \operatorname{rect}\left(\frac{\omega-\omega_{0}}{2 \pi B}\right)
$$

gives a rectangular pulse. 


\subsubsection{Radiation pattern}

The radiation pattern or sonar footprint of a stripmap SAS system maintains the same as it moves along the track. The radiation pattern when the sonar is located at $\mathrm{u}=0$ is denoted by (Soumekh, 1999)

$$
h(\omega, x, y) .
$$

When the sonar is moved to an arbitrary location along the track the radiation pattern will be $h(\omega, x,(y-u))$ which is a shifted version of $h(\omega, x, y)$ in the along-track direction. The radiation experienced at an arbitrary point $(x, y)$ in the spatial domain due to the radiation from the differential element located at $(x, y)=\left(x_{e}(l), y_{e}(l)\right)$ with $l \in S$, where $S$ represents the antenna surface and where the subscript $e$ is used to indicate that it concerns the element location, is,

$$
\begin{aligned}
& \frac{1}{r} i(l) p\left[t-\frac{\sqrt{\left(x-x_{e}(l)\right)^{2}+\left(y-y_{e}(l)\right)^{2}}}{c}\right] d l= \\
& \frac{1}{r} i(l) \exp (i \omega t) \exp \left[-i k \sqrt{\left(x-x_{e}(l)\right)^{2}+\left(y-y_{e}(l)\right)^{2}}\right] d l
\end{aligned}
$$

where $r=\sqrt{x^{2}+y^{2}}$ and $i(l)$ is an amplitude function which represents the relative strength of that element and where the transmitted signal is assumed to be a single tone sinusoid of

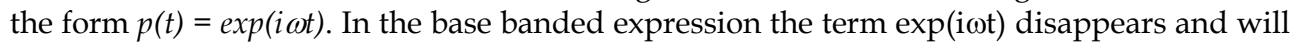
not be considered in the following discussion. The total radiation experienced at the spatial point $(x, y)$, is given by the sum of the radiation from all the differential elements on the surface of the transmitter:

$$
h_{T}(\omega, x, y)=\frac{1}{r} \int_{l \in S} d l i(l) \underbrace{\exp \left(-i k \sqrt{\left(x-x_{e}(l)\right)^{2}+\left(y-y_{e}(l)\right)^{2}}\right)}_{\text {Spherical PM signal }}
$$

Figure. 5. shows the real (blue) and absolute value (red) of $h_{T}(\omega, x, y)$ for a carrier frequency of $f_{0}=50 \mathrm{kHz}$ which corresponds with a radiance frequency $\omega=2 \pi f_{0}$.

The spherical phase-modulated signal $(\mathrm{PM})$ can be rewritten as the following Fourier decomposition,

$$
\begin{aligned}
\exp \left[-i k \sqrt{\left(x-x_{e}(l)\right)^{2}+\left(y-y_{e}(l)\right)^{2}}\right]= \\
\quad \int_{-k}^{k} \exp \left[-i \sqrt{k^{2}-k_{u}^{2}}\left(x-x_{e}(l)\right)-i k_{u}\left(y-y_{e}(l)\right)\right] d k_{u} .
\end{aligned}
$$

By substituting this Fourier decomposition in the expression for $h_{T}$, and after interchanging the order of the integration over $l$ and $k_{u}$, one obtains,

$$
\begin{aligned}
& h_{T}(\omega, x, y)=\frac{1}{r} \int_{-k}^{k} \exp \left(-i \sqrt{k^{2}-k_{u}^{2}} x-i k_{u} y\right) \times \\
& \underbrace{\int_{l \in S} i(l) \exp \left[i \sqrt{k^{2}-k_{u}^{2}} x_{e}(l)+i k_{u} y_{e}(l)\right] d l d k_{u}}_{\text {Amplitude pattern } A_{T}\left(\omega, k_{u}\right)}
\end{aligned}
$$




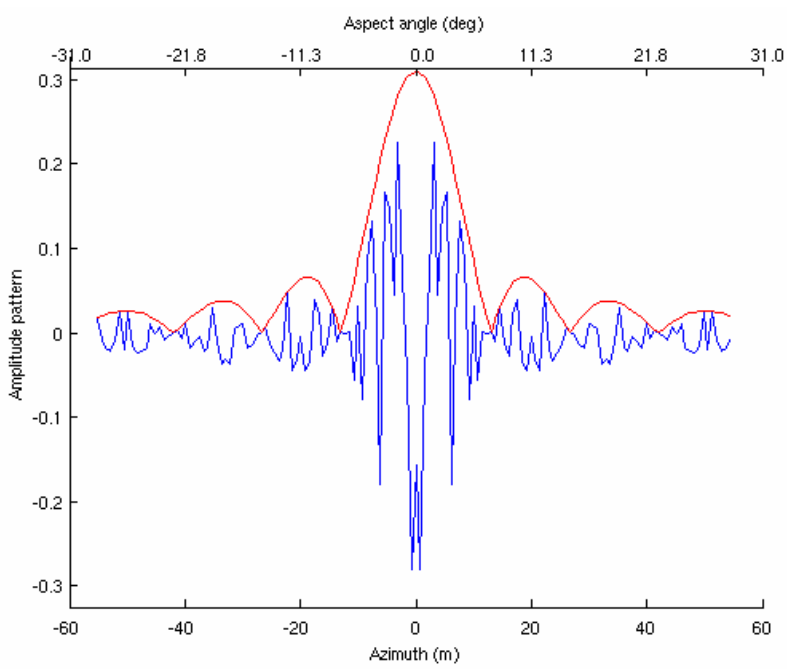

Fig. 5. Total radiation $h_{T}$ experienced at a given point $(x, y)=(100,[-60,60])$ for a given carrier frequency $\mathrm{f}_{0}=50 \mathrm{kHz}$. In blue the real part of $h_{\mathrm{T}}$ is shown, in red the absolute value.

This means that the radiation pattern can be written as an amplitude-modulated (AM) spherical PM,

$$
h_{T}(\omega, x, y)=\frac{1}{r} \int_{-k}^{k} d k_{u} A_{T}\left(\omega, k_{u}\right) \exp \left(-i \sqrt{k^{2}-k_{u}^{2}} x-i k_{u} y\right)
$$

with

$$
A_{T}\left(\omega, k_{u}\right)=\int_{l \in S} i(l) \exp \left[i \sqrt{k^{2}-k_{u}^{2}} x_{e}(l)+i k_{u} y_{e}(l)\right] d l .
$$

The surface for a planar transmitter is identified via,

$$
\left(x_{e}(l), y_{e}(l)\right)=(0, l) \quad \text { for } \quad l \in\left[\frac{-D}{2}, \frac{D}{2}\right],
$$

where $D$ is the diameter of the transmitter. Uniform illumination along the physical aperture is assumed to be, $i(l)=1$ for $l \in\left[\frac{-D}{2} \cdot \frac{D}{2}\right]$ and zero elsewhere. Substituting these specifications in the model for the amplitude pattern $A_{T}$, we obtain,

$$
\begin{aligned}
A_{T}\left(k_{u}\right) & =\int_{-D / 2}^{D / 2} \exp \left(i k_{u}\right) d l \\
& =D \sin c\left(\frac{D k_{u}}{2 \pi}\right)
\end{aligned}
$$

Equation (33) indicates that the transmit mode amplitude pattern of a planar transmitter in the along-track Doppler domain $k_{u}$ is a sinc function that depends only on the size of the transmitter and is invariant in the across-track frequency $\omega$. 


\subsubsection{Motion error implementation}

In an ideal system performance, as the towfish, Autonomous Underwater Vehicle (AUV) or Hull mounted sonar system moves underwater it is assumed to travel in a straight line with a constant along-track speed. However in real environment deviations from this straight along-track are present. By having an exact notion on the motion errors implemented in the simulated data, one can validate the quality of the motion estimation process (section 5).

Since SAS uses time delays to determine the distance to targets, any change in the time delay due to unknown platform movement degrades the resulting image reconstruction. Sway and yaw are the two main motion errors that have a direct effect on the cross-track direction and will be considered here. The sway causes side to side deviations of the platform with respect to the straight path as shown in Fig. 6. This has the effect of shortening or lengthening the overall time-delay from the moment a ping is transmitted to the echo from a target being received. Since, in the case of a multiple receiver system, sway affects all of the receivers equally, the extra time-delay is identical for each receiver. A positive sway makes targets appear closer than they in reality are. In general a combination of two sway errors exist. Firstly the sway at the time of the transmission of the ping and secondly any subsequent sway that occurs before the echo is finally received. Since the sway is measured as a distance with units of meters, we can easily calculate the extra time delay, $\Delta_{\text {sway }}(u)$ given the velocity of sound through water c. The extra time delay for any ping $u$ is,

$$
\Delta t_{\text {sway }}(u)=\frac{X_{T X}(u)+X_{R X}(u)}{c}
$$

where $X_{T X}(u)$ represents the sway at the time of the transmission of the ping under consideration and where $X_{R X}(u)$ represents the sway at the time of the reception of the same ping. Both quantities are expressed in meter. One assumes often that the sway is sufficiently correlated (i.e. slowly changing) so that it is approximately equal in both transmitting and receiving case,

$$
\Delta t_{\text {sway }}(u)=\frac{2 \bar{X}_{\text {sway }}(u)}{c}
$$

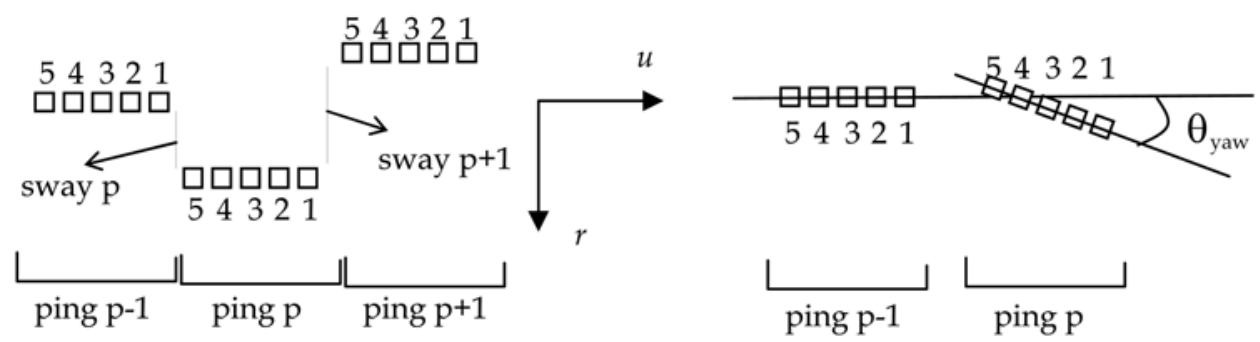

Fig. 6. The effect of sway (left) and yaw (right) on the position of the multiple receivers indicated by the numbers 1 till 5 . The coordinate reference is mentioned between the two representations.

In Fig. 7. one sees the effect on the reconstruction of an image with a non-corrected sway error (middle) and with a corrected sway error in the navigation (right). 

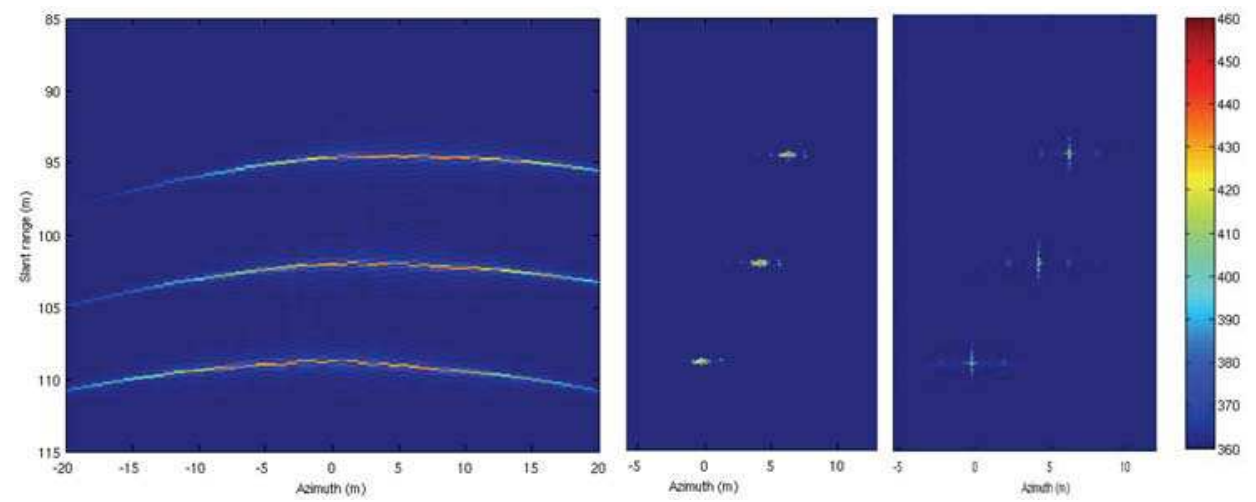

Fig. 7. Echo simulation of 3 point targets with sway error in the motion (left), ( $\omega, \mathrm{k})$-image reconstruction without sway motion compensation (middle) and with sway motion compensation (right).

For sway one has thus the problem of the array horizontally shifting from the straight path but still being parallel to it. With yaw, its effect is a rotated array around the z-axis such that the receivers are no longer parallel to the straight path followed by the platform as illustrated on the right in Fig. 6. Generally speaking there are two yaw errors; firstly when a ping is transmitted and secondly when an echo is received. The examination of those two gives the following; for the case where the transmitter is located at the centre of the rotation of the array, any yaw does not alter the path length. It can safely be ignored, as it does not introduce any timing errors. When the transmitter is positioned in any other location a change in the overall time delay occurs at the presence of yaw. However this change in time delay is common to all the receivers and can be thought of as a fixed residual sway error. This means that the centre of rotation can be considered as collocated with the position of the transmitter.

Yaw changes the position of each hydrophone uniquely. The hydrophones closest to the centre of rotation will move a much smaller distance than those that are further away. The change in the hydrophone position can be calculated through trigonometry with respect to the towfish's centre of rotation. The new position $\overrightarrow{x_{h}}$ ' for each hydrophone $\mathrm{h}$ is given by,

$$
\overrightarrow{x_{h}{ }^{\prime}}=\left(\begin{array}{cc}
\cos \vartheta_{y} & \sin \vartheta_{y} \\
-\sin \vartheta_{y} & \cos \vartheta_{y}
\end{array}\right) \cdot \overrightarrow{x_{h}}
$$

where $\overrightarrow{x_{h}}=(x, y)$ indicates the position of hydrophone $h$ relative to the centre of rotation and $\overrightarrow{x_{h}}{ }^{\prime}=\left(x^{\prime}, y^{\prime}\right)$ indicates the new position of hydrophone $h$ relative to the centre of rotation after rotating around the z-axis due to yaw. $\theta_{\mathrm{y}}$ represents the angle that the array is rotated around the z-axis. For small yaw angles the change in the azimuth $u$ is small and can be ignored. Equation (36) becomes

$$
x_{h}{ }^{\prime}=x_{h}+u_{h} \sin \vartheta_{y} .
$$

Knowing the velocity c of the sound through the medium, one can use equation (37) to determine the change in the time delay $\Delta t_{\text {yaw }}$ i $(u)$ for each hydrophone $h$ 


$$
\Delta t_{\text {yaw }\{h\}}=\frac{\Delta x_{h}^{\prime}}{c}
$$

where $\Delta x_{h}{ }^{\prime}$ represents $x_{h}{ }^{-} x_{h}{ }^{\prime}$ being the cross-track change in position of hydrophone $h$. Fig. 8 . shows the image reconstruction of a prominent point target that has no motion errors in the data compared to one that has been corrupted by a typical yaw.

Once the surge, sway and yaw error vectors are chosen as a function of the ping number, they can be implemented in the simulator as follows;

$$
\begin{gathered}
T X_{r}^{o f f}=t x_{r}^{o} \cos \vartheta_{y}^{p}+t x_{a z}^{0} \sin \vartheta_{y}^{p} \\
T X_{a z}^{o f f}=-t x_{r}^{o} \sin \vartheta_{y}^{p}+t x_{a z}^{0} \cos \vartheta_{y}^{p} \\
R_{\text {out }}=\sqrt{\left(x_{n}-T X_{r}^{o f f}-\operatorname{sway}(p)\right)^{2}+\left(y_{n}-u(p)-T X_{a z}^{o f f}\right)^{2}} \\
R_{\text {back }}=\sqrt{\left(x_{n}-R X_{r}^{o f f}-\operatorname{sway}(p)\right)^{2}+\left(y_{n}-u(p)-R X_{a z}^{o f f}\right)^{2}}
\end{gathered}
$$

Here for a transmitter situated at the centre of the array one can choose the reference system in a way that $t x_{r}{ }^{0}$ and $t x_{a z}{ }^{0}$ are situated at the origin, where the subscript $r$ refers to slant range and $a z$ to the azimuth or the along-track coordinate. Remark that $R_{\text {out }}$ is a scalar whereas $R_{\text {back }}$ is an array $N_{h}$ numbers of hydrophones long.
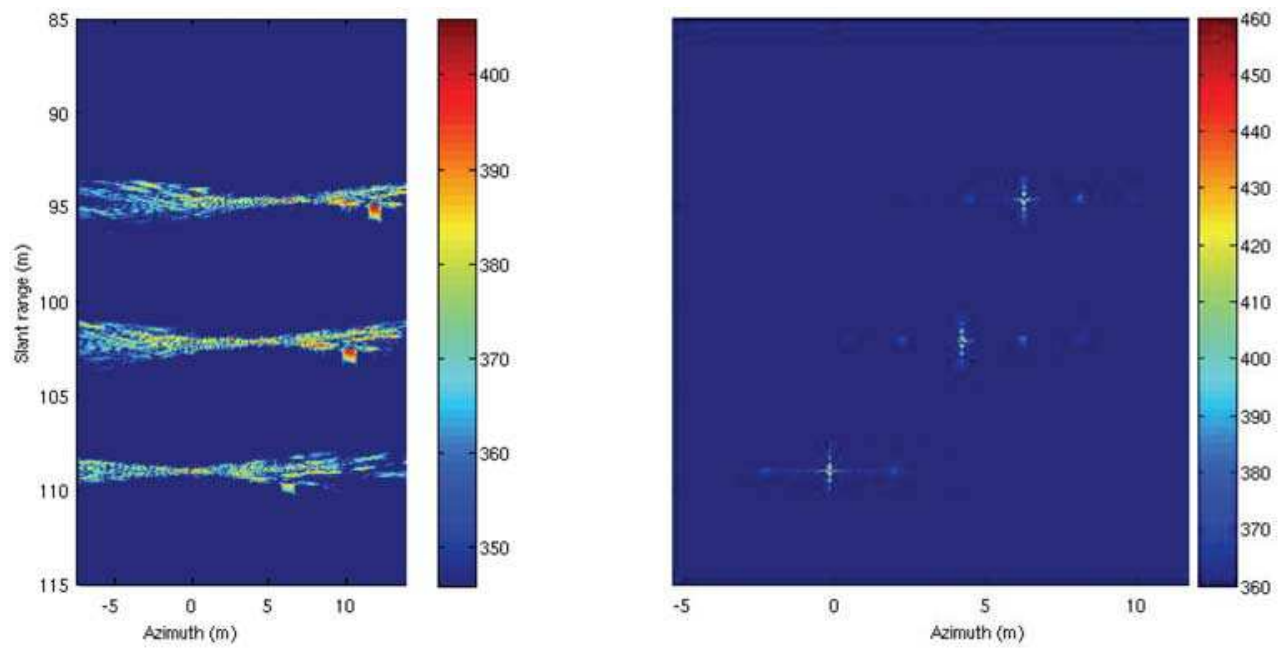

Fig. 8. (w,k)-image reconstruction without yaw motion compensation (left) and with yaw motion compensation (right).

\section{4. $(\omega, k)$ - synthetic aperture sonar reconstruction algorithm}

Form section 3 one studies that a reconstructed SAS image is very sensitive to the motion position and it is necessary to know the position of the sonar at the order of approximately $1 / 10^{\text {th }}$ of the wavelength (a common term to express this is micro navigation). In the 
following sections a brief overview will be given on one particular SAS reconstruction algorithm, i.e. the $(\omega, k)$-reconstruction algorithm (Callow et al. 2001), (Groen, 2006). Afterwards the motion estimation will be explained and finally the motion compensation is illustrated on the $(\omega, k)$-algorithm.

The wave number algorithm, appearing under different names in the literature: seismic migration algorithm, range migration algorithm or $(\omega, k)$-algorithm, and is performed in the twodimensional Fourier transform on either the raw $E E\left(\omega, k_{u}\right)$ or pulse compressed data $S S\left(\omega, k_{u}\right)$. The key to the development of the wave number processor was the derivation of the twodimensional Fourier transform of the system model without needing to make a quadratic approximation. The method of stationary phase leads to,

$$
\mathfrak{I}\left\{\exp \left(-i 2 k \sqrt{x^{2}+(y-u)^{2}}\right)\right\} \cong \sqrt{\frac{\pi x}{i k}} \exp \left(-i \sqrt{4 k^{2}-k_{u}^{2}} \cdot x-i k_{u} \cdot y\right) .
$$

The most efficient way to implement the wave number should be performed on the complex valued base banded data as it represents the smallest data set for both the FFT and the stolt interpolator. It is also recommended that the spectral data stored during the conversion from modulated to base banded is padded with zeros to the next power of two to take advantage of the fast radix-2 FFT. A coordinate transformation also represented by the following stolt mapping operator $S_{b}^{-1}\{$.$\} ,$

$$
\begin{aligned}
& k_{x}\left(\omega, k_{u}\right)=\sqrt{4 k^{2}-k_{u}^{2}}-2 k_{0} \\
& k_{y}\left(\omega, k_{u}\right)=k_{u}
\end{aligned}
$$

The wave number inversion scheme, realizable via a digital processor is than given by,

$$
F F_{b}^{\prime}\left(k_{x}, k_{y}\right)=S_{b}^{-1}\left\{\sqrt{\frac{k}{k_{0}}} \cdot \exp \left[i\left(\sqrt{4 k^{2}-k_{u}^{2}}-2 k\right) \cdot r_{0}\right] \cdot P_{b}^{*}\left(\omega_{b}\right) \cdot E E_{b}^{\prime}\left(\omega_{b}, k_{u}\right)\right\} .
$$

The inverse Stolt mapping of the measured $\left(\omega_{b}, k_{u}\right)$-domain data onto the $\left(k_{x}, k_{y}\right)$-domain is shown in Fig. 9.

The sampled raw data is seen to lie along radii of length $2 k$ in the $\left(k_{x}, k_{y}\right)$-wave number space. The radial extent of this data is controlled by the bandwidth of the transmitted pulse and the along-track extent is controlled by the overall radiation pattern of the real apertures. The inverse Stolt mapping takes these raw radial samples and re-maps them onto a uniform baseband grid in $\left(k_{x}, k_{y}\right)$ appropriate for inverse Fourier transformation via the inverse FFT. This mapping operation is carried out using an interpolation process. The final step is to invert the Fourier domain with a windowing function $W W\left(k_{x}, k_{y}\right)$ to reduce the side lobes in the final image,

$$
\hat{f f}(x, y)=\left|\mathfrak{\Im}_{k_{x}, k_{y}}^{-1}\left\{W W\left(k_{x}, k_{y}\right) \cdot \hat{F F}\left(k_{x}, k_{y}\right)\right\}\right| .
$$

This windowing operation can be split into two parts; data extraction and data weighting. In data extraction the operation first extracts from the curved spectral data a rectangular area of the wave number data. The choice of the 2-D weighting function to be applied to the 


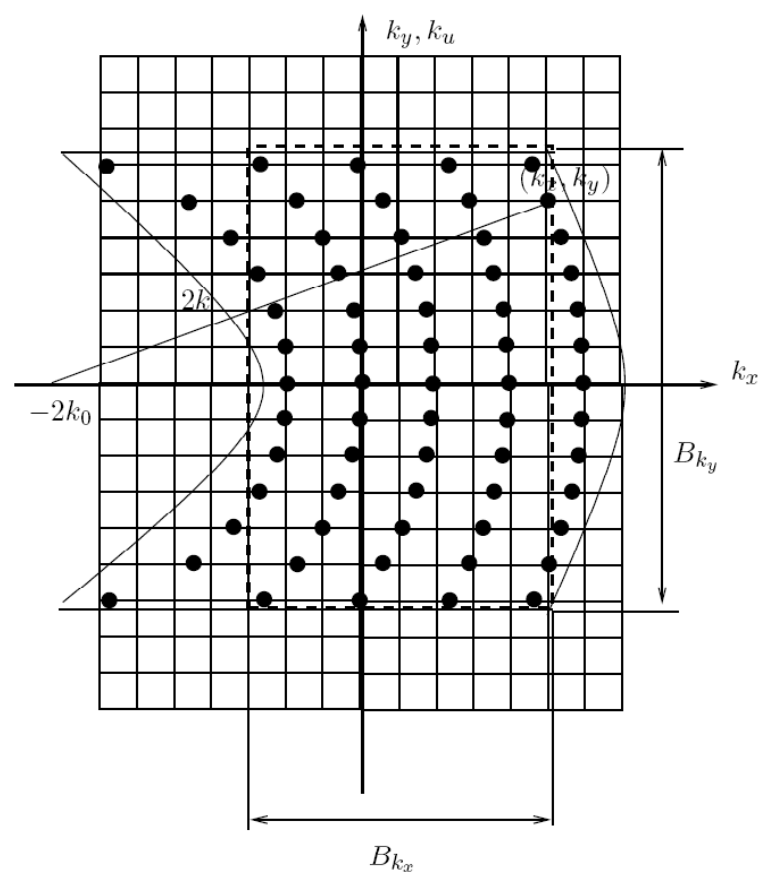

Fig. 9. The 2D collection surface of the wave number data. The black dots indicate the locations of the raw data samples along radii $2 \mathrm{k}$ at height $\mathrm{k}_{\mathrm{u}}$. The underlying rectangular grid shows the format of the samples after mapping (interpolating) to a Cartesian grid $\left(\mathrm{k}_{\mathrm{x}}, \mathrm{k}_{\mathrm{y}}\right)$. the spatial bandwidths $B_{k x}$ and $B_{k y}$ outline the rectangular section of the wave number data that is extracted, windowed and inverse Fourier transformed to produce the image estimate.

extracted data is arbitrary. In the presented case a rectangular window and a 2-D Hamming window is used. Before applying the $k_{y}$ weighting across the processed $3 \mathrm{~dB}$ radiation bandwidth, the amplitude effect of the radiation pattern is deconvoluted as,

$$
W W\left(k_{x}, k_{y}\right) \cdot \operatorname{rect}\left(\frac{k_{y}}{B_{k_{y}}}\right) \cdot \frac{1}{A\left(k_{y}\right)} \cdot W_{h}\left(\frac{k_{x}}{B_{k_{x}}}\right) \cdot W_{h}\left(\frac{k_{y}}{B_{k_{y}}}\right)
$$

where $W_{h}(\alpha)$ is a $1 \mathrm{D}$ Hamming window defined over $\alpha \in[-1 / 2,1 / 2]$ and the wave number bandwidths of the extracted data shown in Fig. 9. are

$$
\begin{aligned}
& B_{k_{x}}=\sqrt{4 k_{\max }^{2}-\left(\frac{2 \pi}{D}\right)^{2}}-2 k_{\min } \approx \frac{4 \pi B_{c}}{c}-\frac{2 \pi^{2}}{k_{\max } D^{2}} \\
& B_{k_{y}}=\frac{4 \pi}{D}
\end{aligned}
$$

here $k_{\min }$ and $k_{\max }$ are the minimum and maximum wave numbers in the transmitted pulse, $B_{c}$ is the pulse bandwidth $(\mathrm{Hz})$ and $D$ is the effective aperture length. The definition of the $x$ - 
and $y$-axes depends on the number of samples obtained within each wave number bandwidth, the sampling spacing chosen, and the amount of zero padding used. The Hamming window over a length $N+1$ is given by its coefficients, which are calculated by,

$$
w_{h}(n)=0.5386-0.46164 \cdot \cos \left(\frac{2 \pi n}{N}\right), \text { with } 0 \leq n \leq N .
$$

The resolution in the final image is given by,

$$
\begin{aligned}
& \delta x_{3 d B}=\frac{2 \pi}{B_{k_{x}}}=\alpha_{w} \cdot \frac{c}{2 B_{e f f}} \\
& \delta y_{3 d B}=\frac{2 \pi}{B_{k_{y}}}=\alpha_{w} \cdot \frac{D}{2}
\end{aligned}
$$

where $\alpha_{\mathrm{w}}=1.30$ for the Hamming window.

\section{Platform motion estimation}

In order to be able to explain properly the functioning of the Displaced Phase Centre Array (DPCA) algorithm (Bellettinit \& Pinto 2000 and 2002) he simulation echoes on 9 targets arranged around the central target were generated. Some a priori known sway and yaw errors were included in the straight line navigated track. The positions of the 9 targets can be seen in Fig. 10 (left) and are given relative to the target that is situated at the center of the scene $(0,0)$. At the right side the corresponding echo is shown. The simulator used an array consisting of 15 hydrophones separated by $\Delta \mathrm{R}_{\mathrm{x}}=21.88 \mathrm{~cm}$. The slant range covered goes
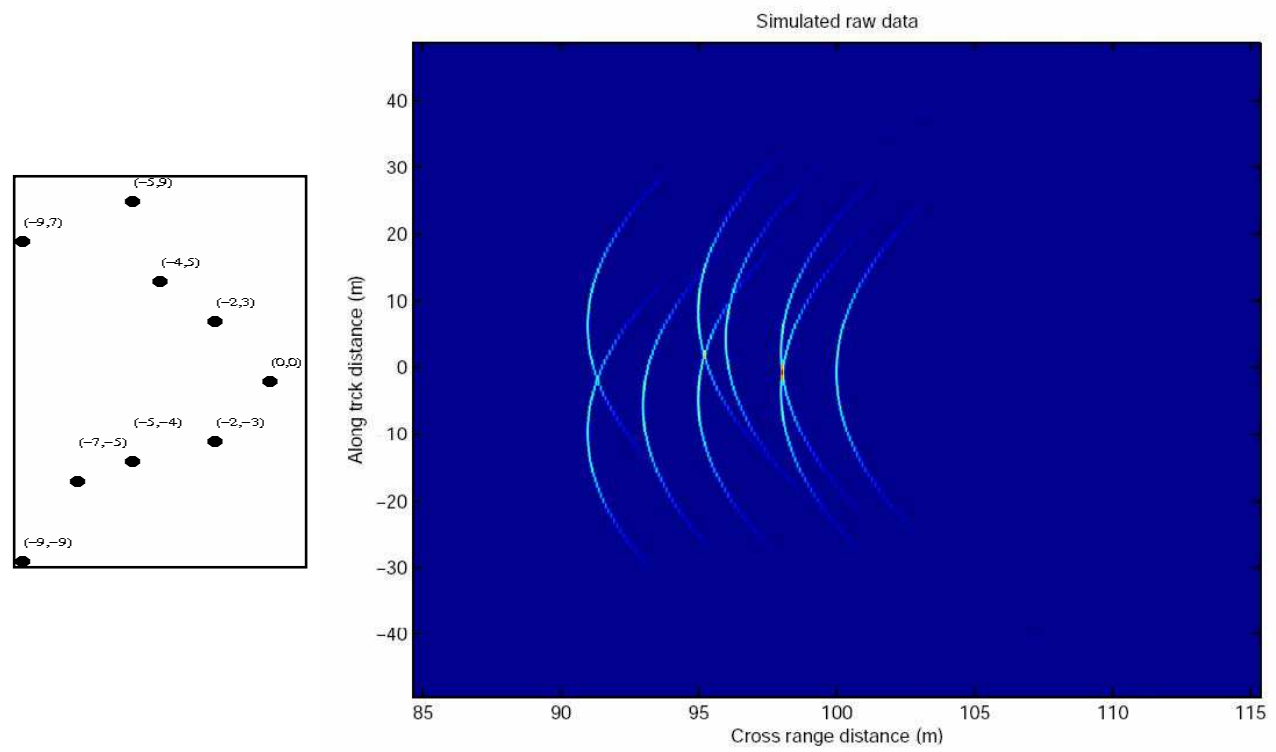

Fig. 10. Target position $(\mathrm{r}, \mathrm{u})$ given relative to the center of the scene at $\mathrm{r}_{0}=100 \mathrm{~m}$. 
from $84.64 \mathrm{~m}$ till $115.33 \mathrm{~m}$. The data shown at the right side of Fig.10 shows only the return observed in receiver number 1 . The echo data for a fixed ping number are presented in Fig. 11. for ping=61 (left) and for ping=62 (right) as function of the 15 hydrophones (x-axis) and the range (y-axis).

One can clearly see around the smallest ranges that the distance to the target is function of the receiver itself. The platform speed was chosen in a way $(v=1.2763 \mathrm{~m} / \mathrm{s})$ that the echoes for receiver 1 till 8 for ping number $n$ should coincide with those of 8 till 15 for ping number $n+1$. This set of 2 times 8 receivers serves as the input data for the dpca-code. The aim of the DPCA-motion compensation method is to estimate from the maximal correlation between the two pings data the surge, sway and yaw.
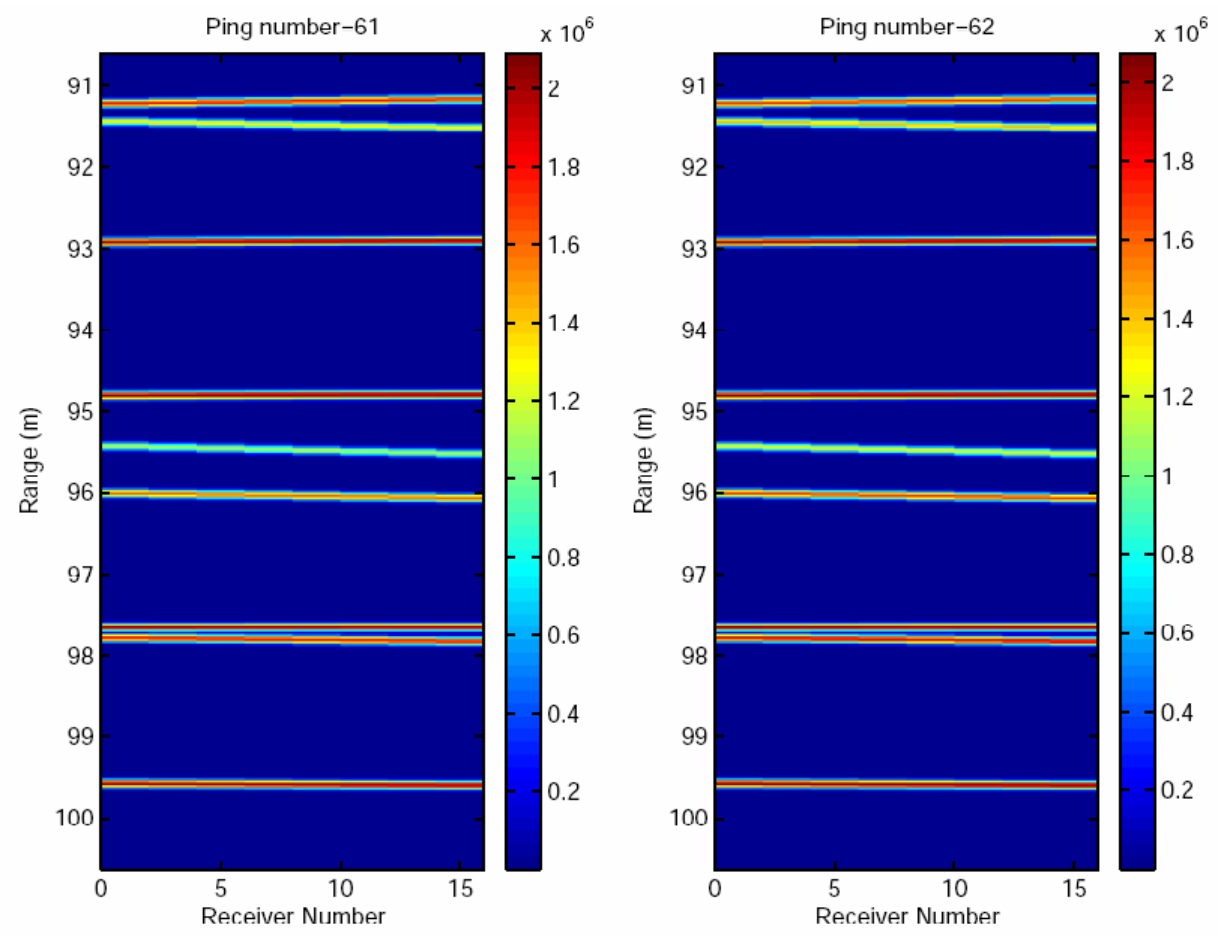

Fig. 11. The echoes are shown for the 15 different receivers as a function of the slant range for (left) ping number 61 and (right) for ping number 62. In this simulation no motion errors were introduced and the speed of the platform $(\mathrm{v}=1.2763 \mathrm{~m} / \mathrm{s})$ was chosen in a way that 8 receivers are overlapping between two consecutive pings.

\subsection{Phase Center Array (PCA) approximation}

In the PCA approach one approximates a true bistatic sonar as a monostatic sonar. On other words one will treat the bistatic transmitter/receiver pair as if it were a single co-located transducer located midway between the two. The error caused in making the phase-center approximation is the difference between the two-way bistatic path and the two-way equivalent monostatic path (Fig. 12). Writing out the approximation error, $\varepsilon$, gives; 


$$
\varepsilon=\sqrt{x^{2}+(u-y)^{2}}+\sqrt{x^{2}+(u+\sigma-y)^{2}}-2 \sqrt{x^{2}+\left(u+\frac{\sigma}{2}-y\right)^{2}}
$$

where $\sigma$ is the relative position of the hydrophone with respect to the transmitter. The series expansion for $\sigma / y<<1$ one obtains

$$
\varepsilon \approx \frac{\sigma^{2}}{4 r} \cos ^{2} \vartheta+\frac{\sigma^{4}}{64 r^{3}} \cos ^{2} \vartheta\left(4-5 \cos ^{2} \vartheta\right)+\ldots
$$

where $\vartheta$ is the bearing of the target with respect to the position of the co-located transducer and $r$ the distance between the co-located transducer and the target. For a far field condition, PCA holds when $\frac{\sigma^{2}}{4 r}<<\lambda_{0}$.

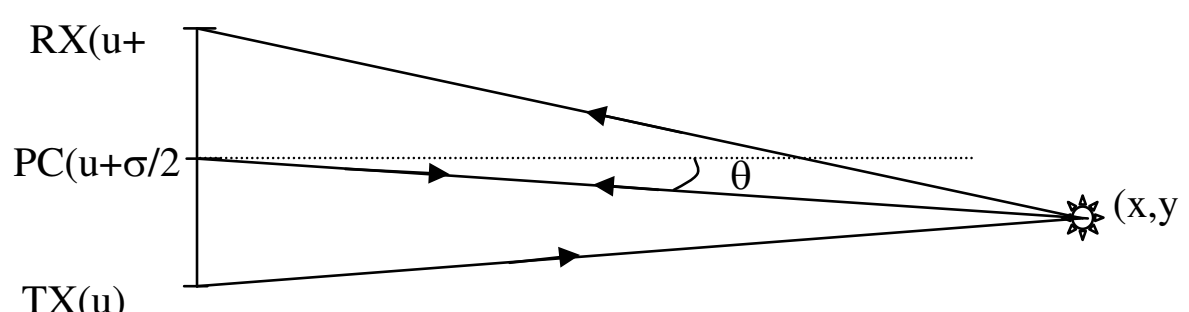

Fig. 12. Geometry of the phase centre approximation. Tx, Rx, PC are respectively the position of the transmitter, the receiver and the phase centre position. The target is located at position $(\mathrm{x}, \mathrm{y})$.

\subsection{Array geometry and platform displacement}

A $1 D$ array consisting of 15 receivers displaced at $\Delta R_{x}=21.88 \mathrm{~cm}$ is used in the simulator. The sonar speed has been chosen to be $v_{8}=1.2763 \mathrm{~m} / \mathrm{s}$ and the pulse repetition time (PRT) to be $T=0.6 \mathrm{~s}$. The transmitter situates in the middle of the receiver array leading to a phase centre array as indicated in Fig. 13. When a higher platform speed is chosen, less overlapping phase centres will exist. This means that for being able to perform a DPCA motion estimation the platform speed will be rather moderate.

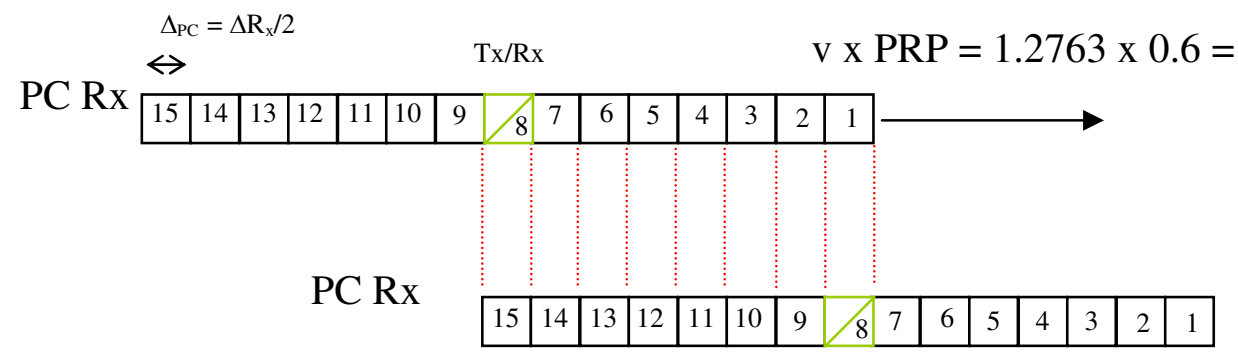

Fig. 13. The number of overlapping phase centers for a 15 hydrophone array with a displacement of $7 \Delta_{\mathrm{PC}}$ leading to 8 overlapping $P C^{\prime}$ s between ping $n$ and ping $n+1$. The 
position of the 15 receivers for ping $n+1$ was shifted for better presentation. In reality PC $\operatorname{Rx}(1)$ of ping $n$ overlaps with $P C \operatorname{Rx}(8)$ of ping $n+1$ etc. The diagonal in $\operatorname{Rx}(8)$ indicates that at this position there is also a transmitter element present.

The following formula can be used to define the platform speed for a given PRT in order to have $n \leq H$, with $H$ the total number of receivers in the array, overlapping phase centres;

$$
v=\frac{(H-n) \Delta_{P C}}{P R T} .
$$

Due to these overlapping phase centres between two consecutive pings, a correlation analysis can be performed to get an idea about the motion errors. Therefore the notion of $x-$ lag (cross-range direction) and t-lag (range direction) has to be introduced.

\subsection{The notion of $x$-lag}

When the platform displacement is chosen like mentioned in section 5.2, eight receivers will overlap for two consecutive pings like shown in Fig. 13. However in real situations the platform motion will deviate from this ideal trajectory. When the speed of the platform will be lower than $v_{8}, R_{x 1}(n)$ will no longer collocate with $R_{x 8}(n+1)$. Here, to indicate the receiver number a subscripted index is used, and the ping number is given between brackets. Assuming that the platform speed was only $v=1.0940 \mathrm{~m} / \mathrm{s}$ instead of $v 8$, than $R_{x 1}(n)$ will collocate with $R_{x 7}(n+1), R_{x 2}(n)$ with $R_{x 8}(n+1), .$. and $R_{x 9}(n)$ with $R_{x 15}(n+1)$. Therefore the $x-l a g$ will be used to determine the along track motion of the platform. Due to the fact that the data volume on which the DPCA will be applied has to be kept as small as possible, one stocks only a limited amount of receiver returns. First of all one makes a mean speed estimation of the platform (most reliable is the autopilot speed of the vessel). Corresponding this speed one knows the approximate overlapping phase centres by the use of equation (50). Only those receiver returns will be considered in the DPCA analysis and corresponds to an $x-l a g=0$. An $x-l a g=+1$ will be executed on $R_{x 2-8}(n)$ and $R_{x 8-14}(n+1)$ as shown in Fig. 14 . In this analysis $5 x$-lags are tested going from $-2,-1,0,1,2$, being sensitive in detecting speed deviations between $\mathrm{v}=1.641 \mathrm{~m} / \mathrm{s}$ for an $\mathrm{x}-\mathrm{lag}=-2$ and $\mathrm{v}=0.9116 \mathrm{~m} / \mathrm{s}$ for an $\mathrm{x}-\mathrm{lag}=+2$.

From Fig. 14 one sees that the correlation for an $x-\operatorname{lag}=-2$ or +2 is performed on 6 receivers for each ping, for an $x$-lag $=-1$ or +1 it is performed on 7 receivers and for an $x$-lag $=0$ it is performed on the eight receivers that were memory stacked.

\subsection{The notion of t-lag}

As mentioned before, the $x$-lag notion refers to the receiver array, while the t-lag notion refers to the range. Therefore, the t-lag will be used to determine the time delay leading to a estimation of the sway and the yaw. In the DPCA analysis, for each x-lag*, a cross correlation is taken between the two consecutive ping data with a chosen t-lag of 8 based on following expression:

$$
c_{f g}^{m}=\sum_{n-\infty}^{\infty} f[n+m] \times g^{*}[n]
$$

\footnotetext{
- Here 5 different $x$-lags are considered, leading to 5 cross correlation plots as shown in Fig. 15.
} 
where $m$ indicates the value of the t-lag (i.e. shift in the slant range between the two vectors $\mathrm{f}$ and $\mathrm{g}$ ), $\mathrm{f}$ and $\mathrm{g}$ are the overlapping Rx couple for respectively ping $n$ and ping $n+1$ and $g^{*}$ represents the complex conjugate of $g$. Therefore, for each overlapping $R x$ couple, one obtains 17 cross correlations if a t-lag of 8 is chosen (Fig. 15). For a t-lag of $8 m$ runs from $-8,-$ $7, \ldots, 0,1, \ldots 7,8$. The cross correlation is normalized in a way that the autocorrelations at zero lag are identically 1.0 .

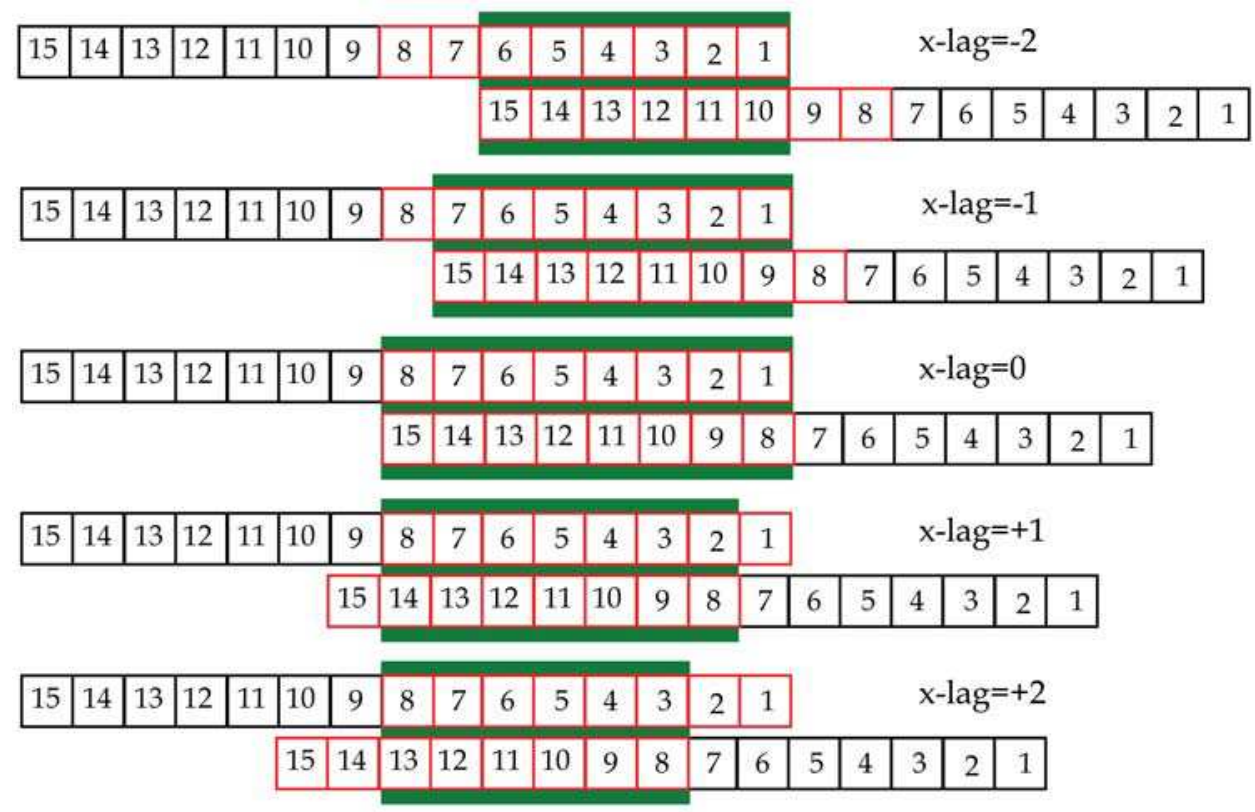

Fig. 14. The overlapping receivers between ping $n$ and $n+1$ for the different $x$-lags under consideration $(-2,-1,0,1,2)$. The receivers indicated with the green bar are the ones that will be cross correlated. The receivers surrounded in red are the ones that are memory stacked during the DPCA processing. Each receiver contains the time series containing the echo information.

Thus for an $x$-lag=-1 the cross correlation takes place between 7 phase centre couples (see Fig. 16.), i.e. $\left(R_{x 1}(p), R_{x 9}(p+1)\right),\left(R_{x 2}(p), R_{x 10}(p+1)\right), \ldots,\left(R_{x 7}(p), R_{x 15}(p+1)\right)$. The $x$-axis in the cross correlation pots represents those couples and is called $R x$ couple id. Remark that an $R x$ couple $i d=1$ for an $x$-lag=-1 corresponds to $\left(R_{x 1}(p), R_{x 9}(p+1)\right)$ whereas an $R x$ couple $i d=1$ for an $x$ lag $=+1$ corresponds to $\left(R_{x 2}(p), R_{x 8}(p+1)\right)$. Remark also that the number of $R x$ couple ids depends on the $x$-lag that is considered. There are 6 couples for $x$-lag $=+2$ or $-2,7$ couples for $x$-lag $=+1$ or -1 and 8 couples for $x$-lag=0. Once the Rx couple id is chosen, (lets take for instance $\left.\left(R_{x 4}(p), R_{x 12}(p+1)\right)\right)$ the slant range vectors are correlated with a y-ordinate given by the chosen t-lag=8. Fig. 17 is an illustration of the $t-l a g=-4,0$ and +4 for an $x-l a g=-1$ and for the first receiver couple id. 

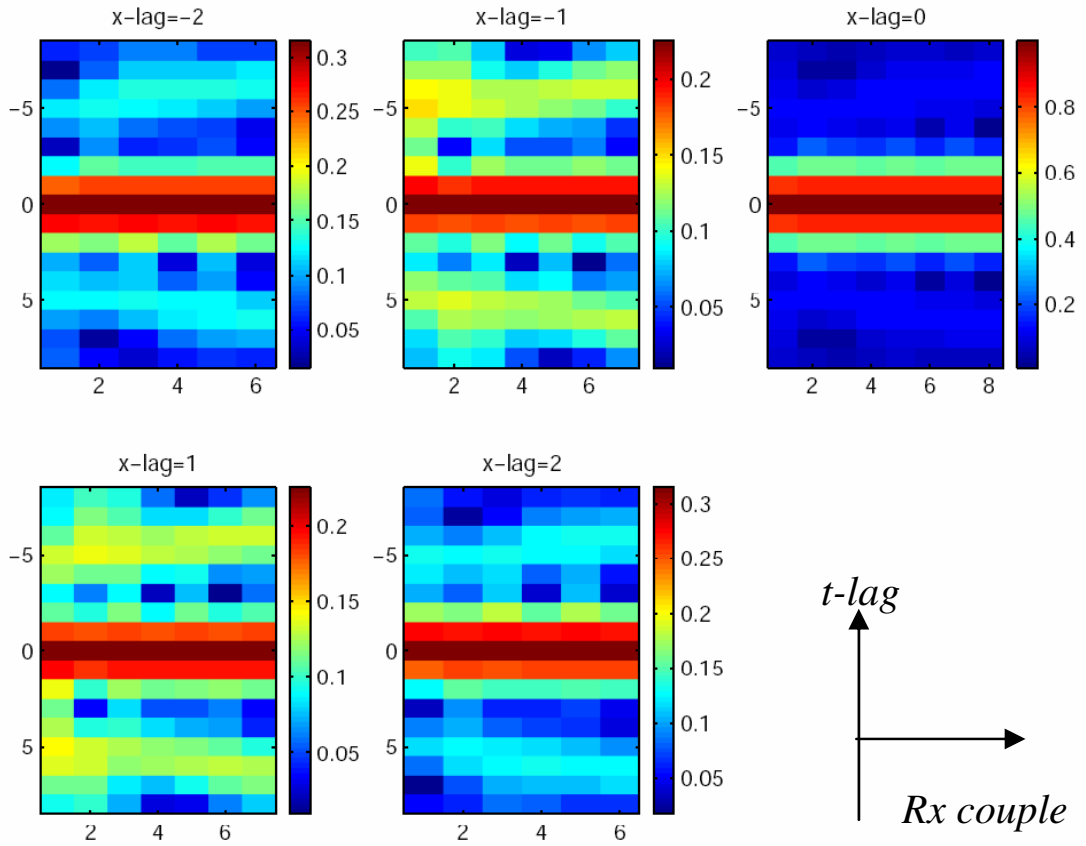

Fig. 15. Cross correlation $\left(c_{f g}^{m}\right)$ with a t-lag of 8 , giving $17 \mathrm{y}$-samples and 6, 7, 8, 7, $6 \mathrm{x}$ samples for the respective $-2,-1,0,1,2$ x-lags.

ping $\mathrm{P}$
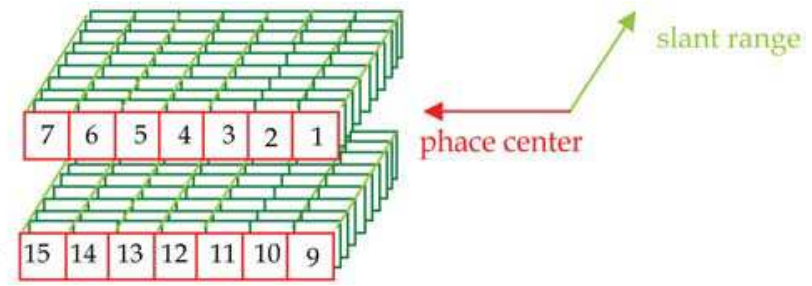

ping $\mathrm{p}+1$

phace center

Fig. 16. (Slant range - phase center)-representation for an $\mathrm{x}-\mathrm{lag}=-1$ between ping $\mathrm{p}$ and $\mathrm{p}+1$. The receiver (i.e. more precisely the phase center corresponding a particular $\mathrm{Rx}$ ) couples are $\left(R_{x 1}(p), R_{x}(p+1)\right),\left(R_{x 2}(p), R_{x 10}(p+1)\right), \ldots,\left(R_{x 7}(p), R_{x 15}(p+1)\right)$.
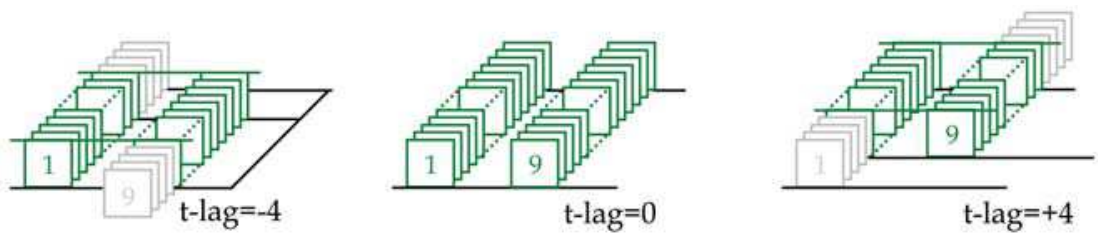

Fig. 17. Respective $t$-lag $=-4,0$ and +4 representation of the $R x$ couple $i d=1$ (i.e. $\left.\left(R_{x 1}(p), R_{x}(p+1)\right)\right)$ for the $x$-lag= -1 . The gray slant range bins will not contribute in the calculation of the cross correlation given in equation (51). 


\subsection{Beam forming on the cross correlation}

To estimate the amplitude and angle of arrival one can perform beam forming on the cross correlation plots. But first, a general theory on beam forming is introduced in order to be able to explain the extension of this theory onto the cross correlation plots. This extension will form the core of the DPCA motion estimation idea.

\subsubsection{Spatial time delay}

Fig. 18. shows a linear array of equally spaced hydrophone elements being intercepted by a propagating wave front at beam angle $\theta$. To make the far field approximation of the top figure one makes the following assumptions;

$$
\begin{aligned}
& \theta_{i} \approx \theta \\
& r_{i} \approx r \quad \text { (for amplitude variations) } \\
& r_{i} \approx r+x_{n} \quad \text { (for phase variations) }
\end{aligned}
$$

with

$$
x_{n}=\left(n-\frac{H+1}{2}\right) d \sin \theta .
$$

The corresponding spatial time delay associated with the distance $x_{n}$ is given by

$$
t_{n}=\frac{x_{n}}{c}
$$

where $\mathrm{c}$ is the velocity of the wave and $\mathrm{n}$ the receiver under consideration. The corresponding spatial phase delay is given by

$$
\phi_{x_{n}}=\frac{2 \pi}{\lambda} x_{n}
$$
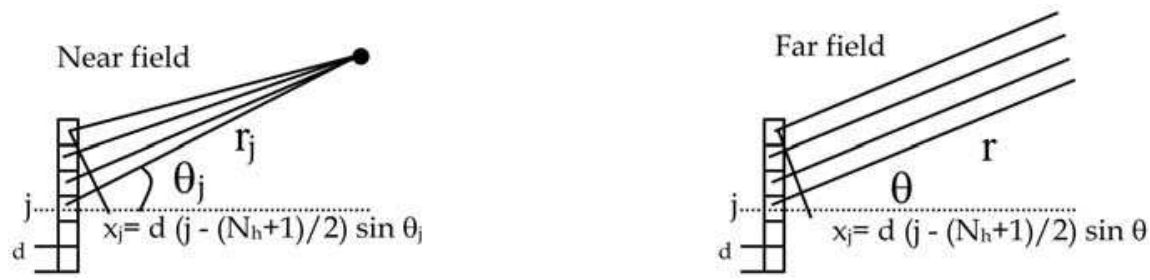

Fig. 18. Equally spaced linear array of hydrophone elements being intercepted by a propagating wave front at beam angles $\theta_{j}$ for the near field configuration (left) with beam angle $\theta$ for the far field (right) approximation.

It is usually more meaningful when discussing array performance to express the phase delays in terms of the carrier frequency $f_{0}$ and the array frequency $f_{a}$. The array frequency is the frequency whose half-wavelength is equal to the inter hydrophone spacing $d$, i.e.

$$
d=\frac{\lambda_{a}}{2}=\frac{c}{2 f_{a}} .
$$


Substituting this and (53) into (55) yields

$$
\phi_{x_{n}}=2 \pi\left(n-\frac{H+1}{2}\right) \frac{\lambda_{a}}{2 \lambda} \sin \theta .
$$

\subsubsection{Beam steering}

An array can be electronically steered by introducing processing phase or time delays into the hydrophone outputs. The processing delay inserted in series with the $\mathrm{n}^{\text {th }}$ element output in order to steer the array at angle $\theta_{0}$ is given by

$$
\phi_{p_{n}}=2 \pi \frac{\Delta r_{n}}{\lambda} \quad \text { with } \Delta r_{n}=n d \sin \theta_{0}
$$

where $\Delta r_{n}$ is the path correction due to the steering of the beam (Fig. 19.). If the steering angle is around the central receiver,

$$
n \in\left[\frac{-H+1}{2}, \frac{-H+1}{2}+1, \ldots, \frac{H-1}{2}\right] .
$$

The beam forming process sums the delayed outputs from the hydrophone elements to generate a beam output voltage. This beam voltage can be written in a normalized form

$$
\begin{gathered}
V_{b}=\frac{1}{N_{h}} \sum_{n=0}^{N_{h}-1} V_{n} \exp \left(-i \phi_{p_{n}}\right) \\
=\frac{1}{N_{h}} \sum_{n=0}^{N_{h}-1} V_{n} \exp (-i n \Delta \phi)
\end{gathered}
$$

where $\Delta \phi=\frac{\pi f}{f_{a}} \sin \theta_{0}$ is the processing phase increment. Let $\theta_{0}(k)$ represent the $\mathrm{k}^{\text {th }}$ beamsteering angle, than

$$
\Delta \phi(k)=\frac{\pi f}{f_{a}} \sin \theta_{0}(k)
$$

represents the processing phase increment associated with the $k^{\text {th }}$ beam-steering angle. Restricting the beam angles $\theta_{0}(k)$ such that

$$
\Delta \phi(k)=\frac{2 \pi}{N_{h}} k
$$

Substituting the value of $\Delta \phi(k)$ into equation (61) yields

$$
V_{b}(k)=\frac{1}{N_{h}} \sum_{n=0}^{N_{h}-1} V_{n} \exp \left(-i n \frac{2 \pi}{N_{h}} k\right)
$$




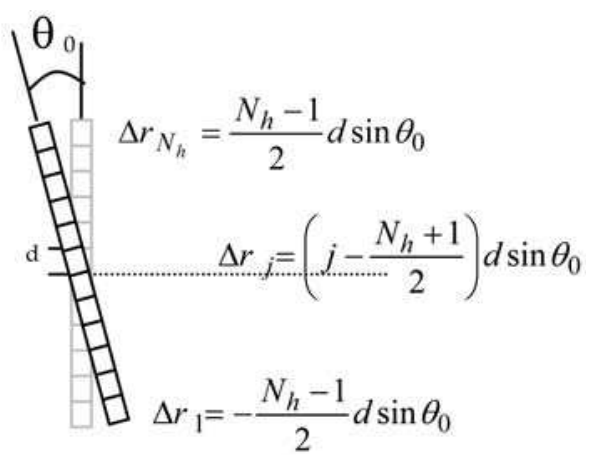

Fig. 19. Steering the multiple hydrophone array at angle $\theta_{0}$.

From equation (62) and (63) one obtains,

$$
\theta_{0}(k)=\sin ^{-1}\left(\frac{2 k}{N_{h}\left(f / f_{a}\right)}\right)=\sin ^{-1}\left(\frac{k c}{L_{h} f}\right)
$$

with

$$
0 \leq k \leq \frac{N_{h}\left(f / f_{a}\right)}{2}=\frac{L_{h} f}{c}
$$

with $L_{h}$ the synthetic aperture over the overlapping $h$ receivers. Equation (64) is used to beamform the cross correlation plots, which leads to a correlation beam. The y-axis indicates still the different $t$-lags, whereas the $x$-axis represents the look angle.
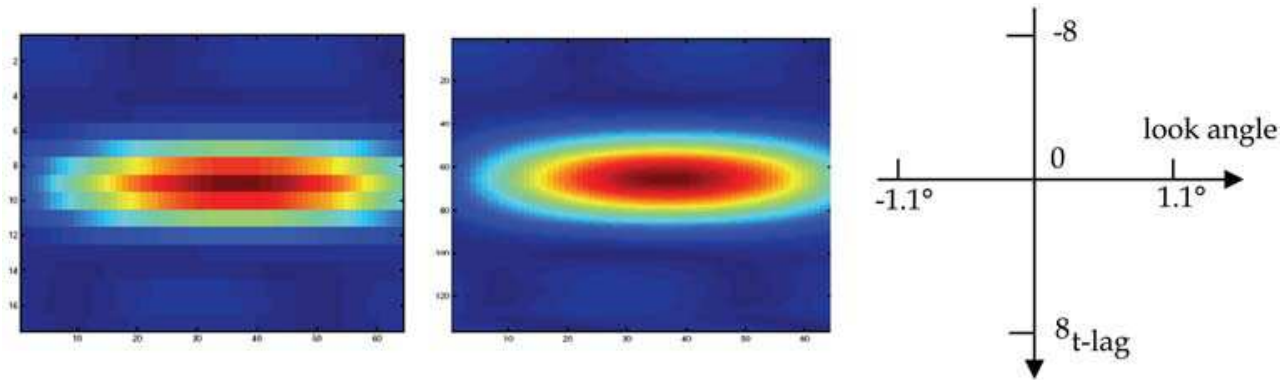

Fig. 20. The beam formed cross correlation matrix before and after interpolation. The interpolation is done along the t-lag with an over sampling factor of 8 , meaning that $17 \mathrm{t}$ lags will become 136 .

A restriction on the look angle is chosen between $\theta=-1.1$ degree till $\theta=1.1$ degree corresponding $\pm \frac{c}{f_{0} L} \frac{180}{\pi}$. The result of the beam forming on one particular cross correlation plot is shown in Fig. 20. In order to find the correlation peaks one has first to smooth the correlation beams using for example a linear interpolation. 


\subsubsection{Correlation peaks and temporal delays}

To find the correlation peaks and the temporal delays as a function of the viewing angle a parabolic maximum finder was designed. For a parabol given by $f(x)=a_{0}+a_{1} x+a_{2} x^{2}$, the refined analytical maximum is given by

$$
f\left(x=-a_{1} /\left(2 a_{2}\right)\right)=\frac{4 a_{0} a_{2}-a_{1}}{4 a_{2}}
$$

Since the 3 point parabolic fit is performed on a normalized dataset, one has to convert the maximum found between -1 and 1 back to its initial scale. For that a simple interpolation is performed. The representation of the 3 points, on which the parabolic fit is performed, is shown by the blue crosses on Fig. 21., the result of the parabolic fit is shown by the green line. The maximum of the parabol is shown by the blue square. One sees a small correction to the integral maximum value towards the new refined maximum.

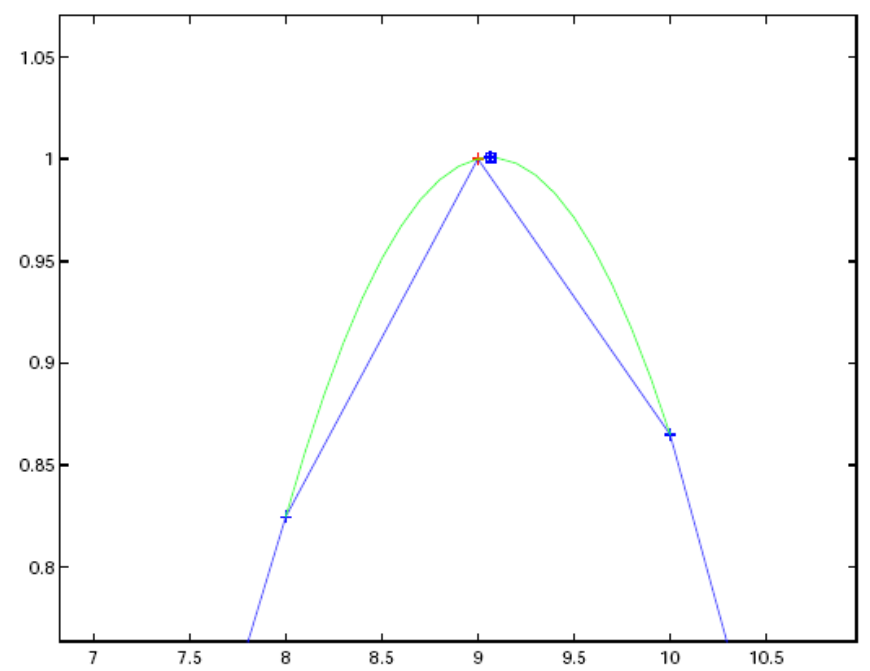

Fig. 21. Illustration of the parabolic maximum finder.

For each $x$-lag (i.e. $-2,-1,0,1,2$ ) the maximum for each look angle is determined on the cross correlation plots after beam forming. Those maxima as function of the look angle are shown in the second line of Fig. 22. The corresponding phase delays are shown in the bottom line of Fig. 22. So for each $x$-lag the best beam is found containing the best beam angle for which the envelope reaches a maximum. Further, the delay that corresponds with the best beam angle is considered the best delay. When the best beam angles are set out as function of the $\mathrm{x}$-lags, the parabolic maximum finder is used to find the corresponding best $\mathrm{x}$-lag. In general this best spatial lag $B_{\operatorname{lag}}(x)$ will not be an integer value but a real number and corresponds to a measure for the surge estimation via,

$$
\text { Surge }=B_{\text {lag }}(x) d / 2 \text {. }
$$


The best lag delay is a measure for the sway estimation via,

$$
\text { Sway }=B_{\text {lag }}(\text { delay }) C / 2 \text {. }
$$

And the best look angle $\theta$ corresponding this best lag is a measure for the yaw estimation via,

$$
\text { Yaw }=B_{\text {lag }}(\theta) \lambda / L
$$
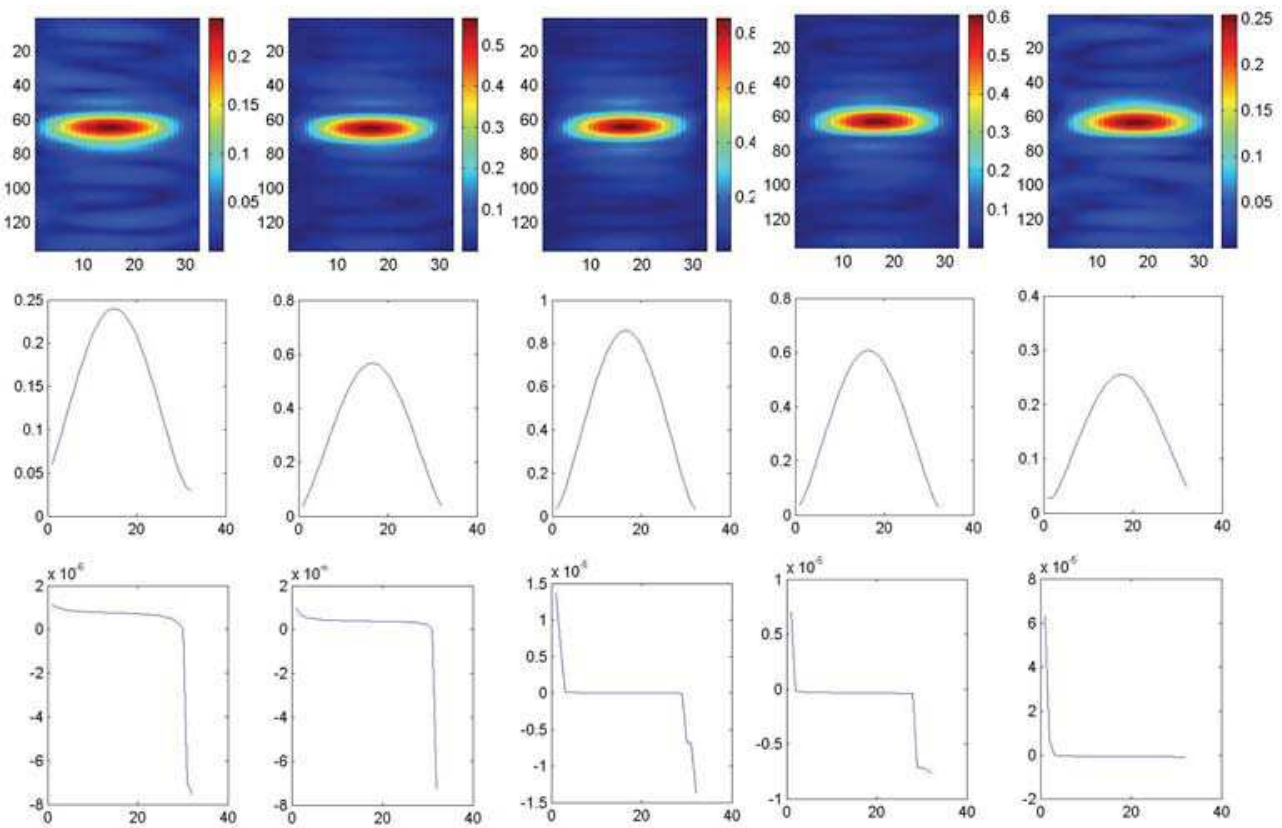

Fig. 22. Each column represents one $x$-lag, going from -2 (utmost left) to +2 (utmost right). The first line represents the cross correlation plots after beam forming. The $x$-axis represents the look angle going from -1.1 till 1.1 degree. For each look angle the 3 point maximum is defined and is shown in the figures at the second line. The third line represents the corresponding phase delays.

For each successive ping-pair the surge, sway and yaw can thus be extracted as is shown in equation (68), (69) and (70). The result of the sway estimation compared to the actual sway that was generated in the simulator is shown on the left-hand side in Fig. 23. The red crosses represent the DPCA sway estimations between a set of different ping-pairs. The line represents the actual generated sway or true sway. On the right-hand side of Fig. 23 the difference is shown between the estimated sway and the true sway expressed in $\mathrm{mm}$. The highest difference between the true and estimated sway is $2 \mathrm{~mm}$, which is well within the $1 / 10^{\text {th }}$ of the applied wavelength $\left(\lambda=3 \mathrm{~cm}\right.$ for a carrier frequency $\left.\mathrm{f}_{0}=50 \mathrm{kHz}\right)$.

Fig. 24 shows the result of the yaw estimation compared to the actual yaw as a function of the ping number (left). The yaw values are expressed in radians. The absolute error between the true and the estimated yaw is of the order of $10^{-4}$ radians (right). 

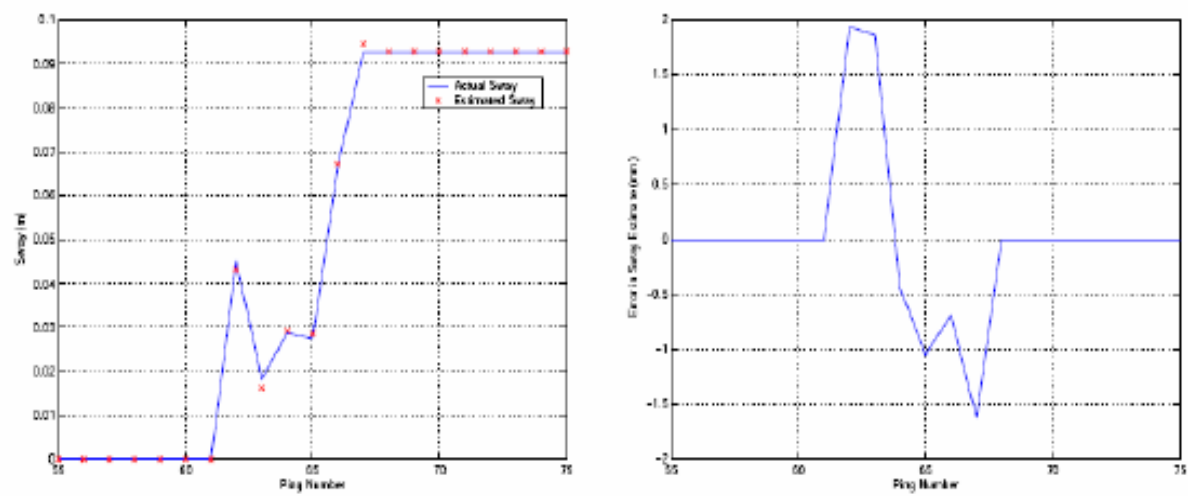

Fig. 23. Result of the sway estimation (red crosses) compared to the simulated sway or actual sway (full line) as a function of the ping number (left). The difference between the actual sway and the estimated sway expressed in $\mathrm{mm}$ (right).
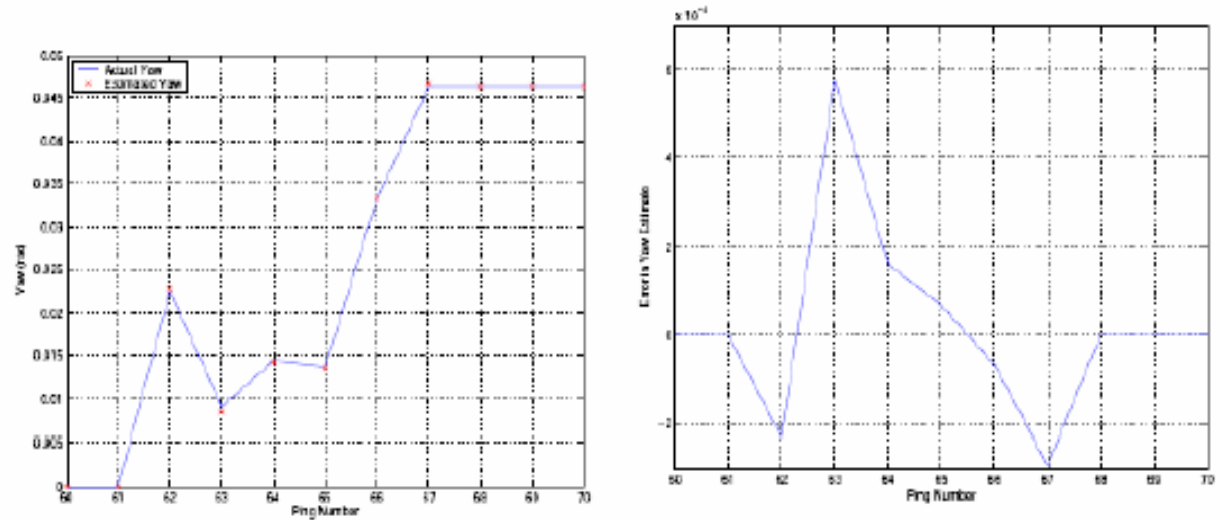

Fig. 24. Result of the yaw estimation (red crosses) compared to the simulated yaw or actual yaw (full line) as a function of the ping number (left). The difference between the actual y and the estimated yaw expressed in deg (right).

\section{Motion correction}

The correction of the surge, sway and yaw motions are done following the estimation of the $\mathrm{x}$-and t-lag analysis obtained in Section 5 .

Let $(O, x, y)$ be the slant range plane (Fig. 23.), with $O x$ the along-track, $O y$ the across-track and $\left(x_{p}, y_{p}\right)$ the coordinates of $C_{p}=\left(T_{p}+R_{p}\right) / 2 . \mathrm{T}_{\mathrm{p}}$ and $\mathrm{R}_{\mathrm{p}}$ are respectively the centres of the real transmitter and receiver position at ping $p$ and $\theta_{p}$ is the angle between $O y$ and the bore-sight to the physical aperture. Than the relative position of the sonar platform can be expressed as, 


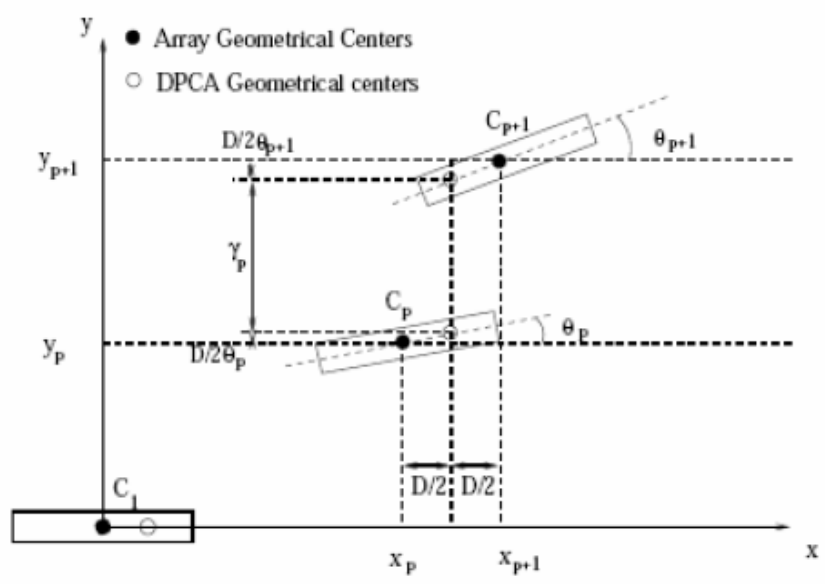

Fig. 23. SAS trajectory representation in the slant range plan

$$
\left\{\begin{array}{l}
x_{p+1}=x_{p}+D \\
y_{p+1}=y_{p}+\gamma_{p}+\frac{D}{2} \theta_{p}+\frac{D}{2} \theta_{p+1} \\
\theta_{p+1}=\theta_{p}+\xi_{p}
\end{array}\right.
$$

where $\gamma_{\mathrm{p}}$ and $\xi_{\mathrm{p}}$ are respectively the DPCA sway and yaw between pings $\mathrm{p}$ and $\mathrm{p}+1$. The angles $\theta_{p}$ have been assumed small (i.e. $\sin \theta \approx \theta$ ). The quantity $\left(y_{p+1}-y_{p}\right)$, which can be interpreted as the physical sway between successive pings, is the sum of three terms. The first is the DPCA sway and the other two result from the heading of the physical reception antenna at ping $\mathrm{p}$ and $\mathrm{p}+1$. The geometrical centre of the DPCA and the one of the physical array are separated by $\mathrm{D} / 2$. This leads to a difference between the real cross-track position and the cross track position of the associated phase centres $\left(D\left(\theta_{p}+\theta_{p+1}\right) / 2\right)$. The estimated trajectory can be expressed as:

$$
\left\{\begin{array}{l}
x_{p}=(p-1) D \\
y_{p}=\sum_{l=1}^{p-1} \gamma_{l}+D \sum_{l=1}^{p-1}\left(p-l-\frac{1}{2}\right) \xi_{p} \\
\theta_{p}=\sum_{l=1}^{p-1} \xi_{l}
\end{array}\right.
$$

The accumulated errors $\delta y_{p}$ and $\delta \xi_{p}$ on the DPCA are given by

$$
\left\{\begin{array}{l}
\delta y_{p}=\sum_{l=1}^{p-1} \delta \gamma_{l}+D \sum_{l=1}^{p-1}\left(p-l-\frac{1}{2}\right) \delta \xi_{l} \\
\delta \theta_{p}=\sum_{l=1}^{p-1} \delta \xi_{l}
\end{array}\right.
$$


The most important effect on SAS processing is the cross track errors. One can see in equation (73) that the along track error depends on the accumulated errors of the DPCA's sway and yaw. In a case where there is only DPCA sway errors $\left(\delta \xi_{p}=0\right)$ they accumulate like a random walk. In a case where there is only DPCA yaw errors $\left(\delta \gamma_{p}=0\right)$ they accumulate like an integrated random walk. In the last case the errors accumulate much faster and lead to a high correlated pattern of phase errors along the SAS.

The differences in cross track as well as in along track positions are leading to a time delay which can be removed by convolving the measured echo with the appropriate delta function $\delta(\mathrm{t}-\Delta \tau)$,

$$
e e_{h}(t, u)=e e_{h}^{r a w}(t, u) \otimes \delta(t-\Delta t) \quad \text { with } \quad \Delta t=\Delta t_{\text {sway }}(u)+\Delta t_{\text {yaw }\{h\}}(u)
$$

where $e e_{h}^{\text {raw }}$ represents the raw data registered at hydrophone $h$ as a function of the delay time $t$ and the azimuth position $u$. $\quad e e_{h}$ represents the motion compensated signal. In practice, instead of performing a convolution, one goes to the frequency domain $(\omega, k)$ using the fast Fourier transform in two dimensions, to perform a simple multiplication,

$$
E E_{h}\left(\omega, k_{u}\right)=E E_{h}^{\prime}\left(\omega, k_{u}\right) \cdot \exp (-i \omega \Delta t) .
$$

\section{Summary}

Synthetic Aperture Sonar (SAS) is a revolutionary underwater imaging technique providing imagery and bathymetry at high spatial resolution with large area coverage. The implementation of synthetic aperture sonar utilising multiple pings to create a virtual long array for range-independent resolution was inadequate due to lack of coherence in the ocean medium, precise platform navigation and high computation rates. Moreover, SAS is far more susceptible to image degradation caused by the actual sensor trajectory deviating from a straight line. Unwanted motion is virtually unavoidable in the sea due to the influence of currents and wave action. In order to construct a perfectly-focused SAS image the motion must either be constrained to within one-tenth of a wavelength over the synthetic aperture or it must be measured with the same degree of accuracy.

The technique known as Displaced phase centers array (DPCA) has proven to be adequate technique in solving the problem of SAS motion compensation. In essence, DPCA refers to the practice of overlapping a portion of the receiver array from one ping (transmission and reception) to the next. The signals observed by this overlapping portion will be identical except for a long track and time shifts proportional to the relative motion between pings. Both shifts estimated by the DPCA are scalars representing the projection of the array receiver locations onto the image slant plane and can be used to compensate for the unwanted platform motion. Thus, the delays observed in the image slant plane can be used to refine the surge, sway and yaw motions.

With advances in innovative motion-compensation, synthetic aperture sonar is now being used in commercial survey and military surveillance systems. Emerging applications for SAS systems include economic exclusion zone mapping (EEZ), mine detection and the development of long range imaging sonar for anti-submarine warfare. 
Although the development of precise navigation sensors and of stable submerged autonomous platforms the motion compensation processing is still a crucial element in the image reconstruction, pre- and/or post-processing.

\section{References}

Bellettini, A. and Pinto, M. A. (2000). Experimental investigation of synthetic aperture sonar Micronavigation, Proceedings of the Fifth ECUA 2000, Lyon, France, (445-450)

Bellettini, A. and Pinto, M. A. (2002). Accuracy of sas micronavigation using a displaced phase centre antenna: theory and experimental validation, Saclantcen report, SR355, $24 \mathrm{p}$.

Bruce, M. P. (1992) A Processing Requirement and Resolution Capability Comparison of Side-Scan and Synthetic-Aperture SOnars, IEEE Journal of Oceanic Engineering, vol. 17, No. 1

Callow, H. J., Hayes, M. P. and Gough, P. T. (2001). Advanced wavenumber domain processing for reconstruction of broad-beam multiple-receiver sa imagery, IVCNZ, (51-56)

Castella, F. R. (1971). Application of one-dimensional holographic techniques to a mapping sonar system. Acoustic Holography, Vol 3.

Christoff, J. T., Loggins, C. D. \& Pipkin, E. L. (1982). Measurement of the temporal phase stability of the medium. J. Acoust. Soc. Am., Vol 71., (1606-1607)

Curlander, J. C. \& McDonough, R. N. (1991). Synthetic Aperture Radar: Systems and Signal Processing, Wiley, ISBN 0-471-85770-X, New York

Cutrona, L. J. (1975). Comparison of sonar system performance achievable using synthetic aperture techniques with the performance achievable by more conventional means. J. Acoust. Soc. Am., Vol 58., (336-348)

Gough, P. T. \& Hayes, M. P. (1989). Measurement of the acoustic phase stability in Loch Linnhe, Scotland. J. Acoust. Soc. Am., Vol 86., (837-839)

Gough, P. T. \& Hawkins, D. W. (1997). Imaging algorithms for synthetic aperture sonar: Minimising the effects of aperture errors and aperture undersampling, IEEE $J$. Oceanic Eng., Vol 22., (27-39)

Groen, J. (2006). Adaptive motion compensation in sonar array processing, PhD thesis, Technical University Delft (TUDelft), Netherlands, $247 \mathrm{p}$.

Hughes, R. G. (1977). Sonar imaging with the synthetic aperture method, Proceedings of the IEEE Oceans, Vol. 9., (102-106)

Marx, D. ; Nelson, M. ; Chang, E. ; Gillespie, W. ; Putney, A. ; Warman, K. (2000). An introduction to synthetic aperture sonar, Proceedings of the Tenth IEEE Workshop on Statistical Signal and Array Processing, pp. 717-721, ISBN: 0-7803-5988-7, Pocono Manor, PA, USA, August 2000

Sherwin, C. W.; Ruina, J. P. \& Rawcliffe, R. D. (1962). Some early developments in syntheti aperture radar systems. IRE Trans. Military Electronics, Vol 6., (111-115)

Skolnik, M. I. (1980). Introduction to Radar Systems, Mc-Graw-Hill, New York

Somers, M. L.; Stubbs A. R. (1984). Sidescan sonar. IEE Proceedings, vol. 131, Part F, no. 3 : (243-256).

Stimson, G. W. (1983). Introduction to Airborn Radar, SciTech, ISBN 1-891121-01-4, New Jersey

Walker, J. L. (1980). Range-doppler imaging of rotating objects. IEEE Trans. Aerospace Electronic Syst, Vol 16., (23-52) 
Walsh, G. M. (1969). Acoustic mapping apparatus. J. Acoust. Soc. Am., Vol 47., (1205)

Wang, L., Bellettini, A., Hollett, R. D., Tesei, A. and Pinto, M.A. (2001). InSAS'00: Interferometric SAS and INS aided SAS imaging, Proc. Oceans'01, Hawaii.

Wiley, C. A. (1985). Synthetic aperture radars. IEEE Trans. Aerospace Electronic Syst, Vol 21., (440-443)

Williams, R. E. (1976). Creating an acoustic synthetic aperture in the ocean. J. Acoust. Soc. Am., Vol 60., (60-73) 


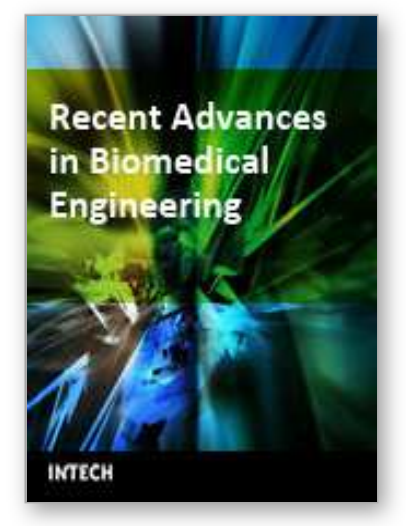

\author{
Advances in Sonar Technology \\ Edited by Sergio Rui Silva
}

ISBN 978-3-902613-48-6

Hard cover, 450 pages

Publisher I-Tech Education and Publishing

Published online 01, February, 2009

Published in print edition February, 2009

The demand to explore the largest and also one of the richest parts of our planet, the advances in signal processing promoted by an exponential growth in computation power and a thorough study of sound propagation in the underwater realm, have lead to remarkable advances in sonar technology in the last years. The work on hand is a sum of knowledge of several authors who contributed in various aspects of sonar technology. This book intends to give a broad overview of the advances in sonar technology of the last years that resulted from the research effort of the authors in both sonar systems and their applications. It is intended for scientist and engineers from a variety of backgrounds and even those that never had contact with sonar technology before will find an easy introduction with the topics and principles exposed here.

\title{
How to reference
}

In order to correctly reference this scholarly work, feel free to copy and paste the following:

R. Heremans, Y. Dupont and M. Acheroy (2009). Motion Compensation in High Resolution Synthetic Aperture Sonar (SAS) Images, Advances in Sonar Technology, Sergio Rui Silva (Ed.), ISBN: 978-3-902613-48-6, InTech, Available from:

http://www.intechopen.com/books/advances_in_sonar_technology/motion_compensation_in_high_resolution_ synthetic_aperture_sonar_sas_images

\section{INTECH}

open science | open minds

\author{
InTech Europe \\ University Campus STeP Ri \\ Slavka Krautzeka 83/A \\ 51000 Rijeka, Croatia \\ Phone: +385 (51) 770447 \\ Fax: +385 (51) 686166 \\ www.intechopen.com
}

\author{
InTech China \\ Unit 405, Office Block, Hotel Equatorial Shanghai \\ No.65, Yan An Road (West), Shanghai, 200040, China \\ 中国上海市延安西路65号上海国际贵都大饭店办公楼405单元 \\ Phone: +86-21-62489820 \\ Fax: $+86-21-62489821$
}


(C) 2009 The Author(s). Licensee IntechOpen. This chapter is distributed under the terms of the Creative Commons Attribution-NonCommercialShareAlike-3.0 License, which permits use, distribution and reproduction for non-commercial purposes, provided the original is properly cited and derivative works building on this content are distributed under the same license. 